\title{
Impact of band-bending on the k-resolved electronic structure of Si-doped GaN
}

\author{
L. L. Lev $\odot,{ }^{1,2,3,{ }^{*}}$ I. O. Maiboroda, ${ }^{4}$ E. S. Grichuk, ${ }^{4}$ N. K. Chumakov, ${ }^{4}$ N. B. M. Schröter, ${ }^{1}$ M.-A. Husanu, ${ }^{1,5}$ T. Schmitt, \\ G. Aeppli, ${ }^{1,6,7}$ M. L. Zanaveskin, ${ }^{4}$ V. G. Valeyev ${ }^{\circ},{ }^{4}$ and V. N. Strocov ${ }^{1, \dagger}$ \\ ${ }^{1}$ Swiss Light Source, Paul Scherrer Institute, 5232 Villigen-PSI, Switzerland \\ ${ }^{2}$ Moscow Institute of Physics and Technology, 9 Institutskiy lane, Dolgoprudny, Moscow Region 141701, Russia \\ ${ }^{3}$ P. N. Lebedev Physical Institute of the Russian Academy of Sciences, 53 Leninskiy Prospekt, 119991 Moscow, Russia \\ ${ }^{4}$ National Research Center "Kurchatov Institute”, 1, Akademika Kurchatova pl., Moscow 123182, Russia \\ ${ }^{5}$ National Institute of Materials Physics, Atomistilor 405A, RO-077125 Magurele, Romania \\ ${ }^{6}$ Department of Physics, ETH Zürich, 8093 Zürich, Switzerland \\ ${ }^{7}$ Institut de Physique, École polytechnique fédérale de Lausanne (EPFL), 1015 Lausanne, Switzerland
}

(Received 9 July 2021; accepted 20 January 2022; published 4 March 2022)

\begin{abstract}
Band bending at semiconductor surfaces and interfaces is the key to applications ranging from classical transistors to topological quantum computing. A semiconductor particularly important for optical as well as microwave devices is GaN. What makes the material useful is not only its large bandgap but also that it can be heavily doped to become metallic. Here, we apply soft-x-ray angle-resolved photoelectron spectroscopy (ARPES) to metallic Si-doped GaN to explore the electron density and momentum-resolved band dispersions of the valence and conduction electrons varying through the surface band-bending region. We find an upward band bending, where the measured band occupation reduces toward the surface, as probed with low photon energies $<0.5 \mathrm{keV}$. The band occupation approaches the bulk value, matching Hall effect measurements, as the photon energy increases to $>1.4 \mathrm{keV}$, where the photoelectron mean free path exceeds the spatial extent of the band-bending region. Our quantitative analysis of the experimental data describes the potential variation in the band-bending region via self-consistent Poisson-Schrödinger equations. We put forward an insightful model to simulate the ARPES spectra from this region through summing up the contribution from all atomic layers, weighted by the photoelectron mean free path, under in-phase conditions achieved at particular values of the photoelectron out-of-plane momentum. The model adequately describes the peculiarities of the ARPES spectra caused by the surface band bending, including the photon-energy dependence of the apparent band occupation and Fermi-surface area, and allows accurate determination of the band-bending profile and values of the photoelectron mean free path. Finally, comparison of our data with supercell density functional theory calculations reveals the preferential location of $\mathrm{Si}$ atoms as substitutional for $\mathrm{Ga}$, with the doped electrons entering the $\mathrm{GaN}$ conduction bands without formation of separate impurity states as would occur for Si interstitials. Our theoretical and experimental results resolve fundamental questions underpinning device performance of the GaN-based and other semiconductor materials in general and demonstrate a general methodology for quantitative studies of electron states in the band-bending region.
\end{abstract}

DOI: 10.1103/PhysRevResearch.4.013183

\section{INTRODUCTION}

Heavily doped $\mathrm{GaN}$ : $\mathrm{Si}$ has a growing number of applications in modern micro- and optoelectronics. Among them are $\mathrm{GaN}$-based high electron mobility transistors (GaNHEMTs) which are key enablers for $5 \mathrm{G}$ telecommunications. Heavily doped $\mathrm{GaN}: \mathrm{Si}$ is employed in such devices for nonalloyed ohmic contacts, with reduced parasitic losses

\footnotetext{
*Corresponding author: 1l1_ru@mail.ru

†Corresponding author: vladimir.strocov@psi.ch
}

Published by the American Physical Society under the terms of the Creative Commons Attribution 4.0 International license. Further distribution of this work must maintain attribution to the author(s) and the published article's title, journal citation, and DOI. and improved reliability compared with conventional alloyed contacts. Preferable approach to form such contacts is the selective deposition of $\mathrm{GaN}: \mathrm{Si}[1,2]$. This approach has several advantages, making it possible to achieve a contact resistance of $<0.2 \Omega \mathrm{mm}$. For this purpose, however, GaN : Si must be heavily doped to a concentration of the dopant atoms $\left(N_{\mathrm{D}}\right)$ of $10^{19} \mathrm{~cm}^{-3}$ and higher. Since in our case the carrier compensation effects [3] are negligible, the conduction electron concentration in the bulk $\left(n_{e}\right)$ determined by the Hall measurements is nearly equal to $N_{\mathrm{D}}$.

Considerable progress has been made in the understanding of physical properties of heavily doped semiconductors (HDSCs) such as GaN : Si during the last 30 years (see, for example, the classic review [4] and an immense number of works citing it). These materials undergo a metal-insulator transition (MIT) where heavy doping drives them into a metallic state. According to a picture of the pair electron-electron 
$(e-e)$ repulsion screened within the Thomas-Fermi model, initially proposed by Mott [5,6], this transition is observed when $N_{\mathrm{D}}$ exceeds a certain critical value estimated as $N_{\mathrm{D}}{ }^{1 / 3} a_{0} \approx$ 0.2 , where $a_{0}$ is the Bohr radius of the donor atom in the lattice. The MIT in $\mathrm{GaN}: \mathrm{Si}$ was studied, for example, in Refs. [7,8] for $n_{e}$ varying between $10^{17}$ and $10^{19} \mathrm{~cm}^{-3}$. In Ref. [7], the electron spin resonance and Hall effect data were analyzed within Shklovskii's formalism [4]. In that work, two Si-related subbands were identified which formed when increasing $n_{e}$ : the $D^{0}$ band of single-occupied Si donor sites and the $D^{-}$band of double-occupied Si states, centered at 27 and $2.7 \mathrm{meV}$, respectively, below the conduction band (CB) minimum (CBM) of GaN. The MIT, ascribed to the Mott mechanism, was found to occur at a critical uncompensated donor $N_{\mathrm{D}}$ of $\sim 1.6 \times 10^{18} \mathrm{~cm}^{-3}$. It was associated with simultaneous closing of the gap between the two subbands and vanishing of the electron transport activation energy in the $\mathrm{CB}$ of $\mathrm{GaN}$. Another work [8] on $\mathrm{GaN}$ : Si has, however, found a different critical $N_{\mathrm{D}}$ value of $2.5 \times 10^{18} \mathrm{~cm}^{-3}$ corresponding to the formation of an impurity band and a value of $2 \times 10^{19} \mathrm{~cm}^{-3}$ leading to its overlap with the CB.

Despite this extensive research, some technologically decisive properties of the HDSCs remain obscure, particularly for extremely large dopant concentrations on the atomic-percent level. Although numerical simulations of devices such as GaN-HEMTs with GaN:Si electrodes routinely employ a model of a host semiconductor with an impurity level of the dopant atoms in the bandgap, the complexity of these materials goes far beyond this simplistic approach. The increase of $n_{e}$ into the heavy-doping regime introduces substantial changes in the host band structure, including a complex interplay between the band filling and gap renormalization known as the Burstein-Moss effect $[9,10]$, effects of the Coulomb interaction and disorder [4], etc. Furthermore, in many cases including $\mathrm{GaN}: \mathrm{Si}$, even the exact position of the dopant atoms in the host lattice stays less known and can hardly be resolved by the standard x-ray and neutron structural analysis methods [11,12] which may be quite case dependent. However, the difference between the interstitial and substitutional positions is decisive for whether the dopant atoms form impurity states decoupled from the host electron system or completely integrate into it and only affect the band filling. Another factor still escaping detailed understanding is a significant band bending near the surfaces and interfaces of the doped semiconductors, which is one of the key factors determining the electrical and optical functionality of their interfaces (see Refs. [13,14] and references therein). For example, the Ga-terminated surfaces of $n$-doped $\mathrm{GaN}$ such as GaN : Si typically exhibit an upward band bending, with the concomitant band bending challenging the ohmiccontact functionality of $\mathrm{GaN}$ : $\mathrm{Si}[15,16]$. This band bending is caused by the negative bound polarization charge at the terminating Ga layer, with the system recovering its charge neutrality $[15,17]$ by generating a variety of compensating electron states including internal ionized states, various surface states originating from the dangling bonds [18], surface vacancies, structural defects, adsorbates or surface oxides [19], etc., which significantly affect the technologically relevant properties of these materials. Another less-known factor that can affect electronic structure of the HDSC interfaces is a spatial variation of the local doping level [20]. All these electron-structure and structural factors strongly complicate a formal description of the HDSCs as well as their surfaces and interfaces, calling for their in-depth experimental studies.

Most experimental results on electronic structure HDSCs have been achieved with magnetoresistance (Hall effect) measurements, optical spectroscopy [7,21], electron spin resonance [8], and other quite indirect analytical methods. A major source of direct information on the bulk, surface, and interface electronic structure resolved in electron momentum (k) is angle-resolved photoemission spectroscopy (ARPES) with variable excitation energy (for entries see, for example, the review [22]). Pushing the ARPES experiments into the soft-X-ray photon-energy range (SX-ARPES) enhances the photoelectron mean free path $\left(\lambda_{\mathrm{PE}}\right)$ by a factor of 3-5 compared with the conventional experiments in the VUV energy range, which is essential to penetrate through potentially distorted surface layers toward the true bulk properties of materials [23-25]. Furthermore, the increase of $\lambda_{\mathrm{PE}}$ translates into high intrinsic resolution of the SX-ARPES experiment in the surface-normal electron momentum $\Delta k_{z}=$ $1 / \lambda_{\mathrm{PE}}[24,26]$, which is crucial for accurate measurements of three-dimensional (3D) electronic dispersions like in GaN. Successful applications of SX-ARPES to extract $\mathbf{k}$-resolved band dispersions in HDSCs have recently been demonstrated, for example, for Mn-doped GaAs [27], Mn-doped InAs quantum dots in GaAs [28], and Fe-doped InAs [29], all potential components of spintronic devices. All previous studies of the band bending [15-17,30-37], however, utilized the X-ray photoelectron spectroscopy (XPS) regime of the photoemission (PE) experiment [38], which implies intrinsic k integration. For example, a recent XPS study on the Ga-polar $n$-type GaN surfaces [16] evaluated the energy position of valence band (VB) maximum (VBM) as a function of depth. In that work, the data analysis was based on the core level (CL)-to-VBM energy difference [30], where hybrid density functional theory (DFT) calculations were used to extract the VBM energy by fitting the VB spectrum. Another study [30,31] evaluated the surface band bending in GaN by fitting the Ga $3 d$ CL spectra at different emission angles. There, the band-bending electrostatic potential $V(z)$ for moderate doping was described with a linear approximation, and for heavy doping, when the extension of $V(z)$ became comparable with $\lambda_{\mathrm{PE}}$, with a quadratic approximation. In the study [32], the surface band bending of $n$-type GaN was probed by means of hard x-ray photoelectron spectroscopy combined with x-ray total reflection, where a steep change of the x-ray attenuation length around the total external reflection was used to control the probing depth from $\sim 2$ to $12 \mathrm{~nm}$.

Here, we present a synchrotron-radiation SX-ARPES investigation of the k-resolved electronic structure of $\mathrm{GaN}: \mathrm{Si}$ in a wide range of technically relevant doping levels above the MIT. These experiments are supported by high-sensitivity Hall measurements as well as first-principles DFT calculations, where the $\mathrm{Si}$ impurities are introduced within the supercell approach. By gradually increasing photon energy (hv) and thus the probing depth of the ARPES experiment, we follow the evolution of electron states through the surface band-bending region toward the bulk. This information about electronic structure in the band-bending region is 

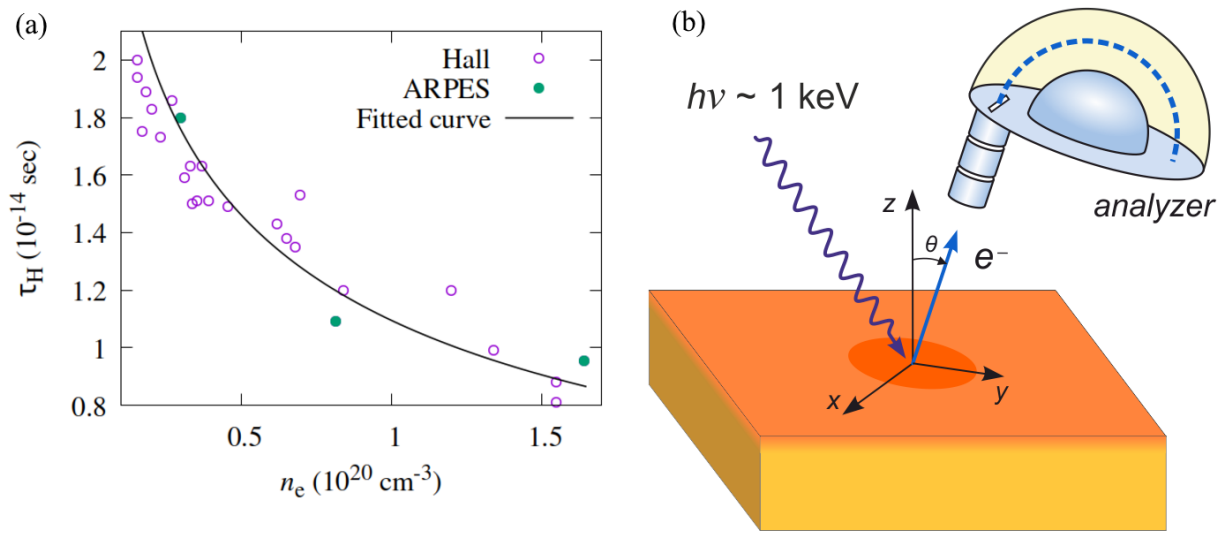

(c)

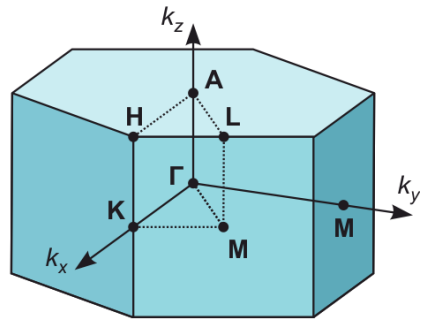

FIG. 1. Soft-X-ray angle-resolved photoelectron spectroscopy (SX-ARPES) experiment on the GaN-based high electron mobility transistors (GaN-HEMT) heterostructure. (a) Electron lifetime $\tau_{H}$ at $E_{F}$ derived from the Hall measurements on a series of samples with different $n_{e}$. The solid green points mark the samples investigated by ARPES (Table I). The solid line shows the fit describing the elastic defect scattering based on Ref. [39]. (b) Scheme of the epitaxial GaN : Si samples investigated by SX-ARPES. The depletion layer on the top of GaN : Si samples is marked by gradient orange color. The photoelectron analyzer detects the distribution $I_{\mathrm{PE}}\left(E_{k}, \vartheta\right)$ of the photoelectron kinetic energy $E_{k}$ and emission angle $\vartheta$, which yield the binding energy $E_{B}$ and momentum $\mathbf{k}$ back in the sample (corrected for the photon momentum $p=h v / c$ ) to produce the sought-for electron dispersions $E(\mathbf{k})$. (c) Bulk Brillouin zone (BZ) of GaN.

essential for the ohmic-contact functionality of $\mathrm{GaN}$ : Si. We develop a theoretical model of the band-bending effect on the k-resolved spectral response of electron states, which describes the puzzling photon-energy dependence of the Fermi momentum apparent in the ARPES spectra. This model links the ARPES spectra with the evolution of bulk electron states through the band-bending region. The achieved results, including the reduced near-surface conduction electron density and the interstitial vs substitution position of the Si dopant atoms, provide essential physical information for theoretical modeling of high-power GaN-based devices with $\mathrm{GaN}: \mathrm{Si}$ contacts, required for further development of nitride electronics.

\section{EXPERIMENTAL PROCEDURE AND RESULTS}

\section{A. Sample growth and characterization}

The samples for our ARPES experiments were epitaxial films of GaN heavily doped with different concentrations of Si impurities. The doped 200-nm-thick GaN layer was grown on an insulating GaN substrate using molecular-beam epitaxy, both oriented in the [0001] crystallographic direction. The Si atoms were introduced during the growth process using a $\mathrm{Si}$ effusion cell. The Si concentration was estimated through Hall measurements. For the ARPES measurements, we used samples with four different $n_{e}$ values of $3.0 \times 10^{19}, 6.4 \times 10^{19}$, $8.3 \times 10^{19}$, and $1.5 \times 10^{20} \mathrm{~cm}^{-3}$. To eliminate surface oxide before the measurements, sample preparation involved chemical treatment in buffered $\mathrm{HCl}$ followed by in situ annealing at a temperature of $\sim 500{ }^{\circ} \mathrm{C}$ for $30 \mathrm{~min}$ (low enough to avoid affecting the dopant distribution profile).

Magnetotransport characterization was carried out in magnetic fields of up to $4 \mathrm{~T}$ applied perpendicular to the sample plane and in the temperature range 4.2-300 K. The dependences of the magnetoresistance tensor components on the temperature and magnetic field were measured in the usual Hall and van der Pauw geometries. The experimental $n_{e}$ varied by $<\sim 3 \%$ through the whole studied temperature range for all samples, confirming their high quality.

The experimental electron mobility values found from the Hall measurements $\left(\mu_{H}\right)$ were used to evaluate the electron lifetimes $\left(\tau_{H}\right)$ at $E_{F}$ as $\tau_{H}=\mu_{H} m^{*} / e$, where $m^{*}$ is the electron effective mass and $e$ the electron charge. These results, obtained for a large series of samples including those used in our ARPES experiments, are presented in Fig. 1(a). Since for the weakly correlated Fermi liquids the $e-e$ interaction at $E_{F}$ is indistinguishable from zero even at elevated temperatures, $\tau_{H}$ reflects only the defect scattering (phonon scattering is much smaller at our sample temperatures). The decrease of $\tau_{H}$ with $n_{e}$ reflects the increase of the defect scattering with doping (the screening of the ionized impurity centers is less significant at the actual doping levels). The experimental points were fitted with the theoretical dependence of the (quasi)-elastic scattering time according to eq. (4) from Ref. [39] (also see Refs. [40,41]). The fitting parameter was the compensation parameter $\theta=\left(N_{\mathrm{D}}-n_{e}\right) / N_{\mathrm{D}}$, which expresses the fraction of electrons localized on fluctuations of the disorder potential and contributing to the tails of density of states (DOS) extending deep into the mobility gap [4]. The fitting was weighted with the average distance of each point to its two nearest neighbors, which accounted for the uneven density of the experimental points along $n_{e}$. The best fit, shown in Fig. 1(a) as the solid curve, corresponds to $\theta=0.072 \pm 0.011$, i.e., nearly all dopant atoms donate conduction electrons.

\section{B. ARPES experiment}

The experiments were performed at the SX-ARPES endstation [24] of the ADvanced RESonant Spectroscopies (ADRESS) beamline [42] of the Swiss Light Source (SLS). The enhanced probing depth of SX-ARPES was essential for our experiments on $\mathrm{GaN}: \mathrm{Si}$ with its surface band-bending region. A high flux of $>10^{13}$ photons/s/0.01\%BW delivered by the beamline/undulator combination was essential for our experiments in the SX range where the photoexcitation 

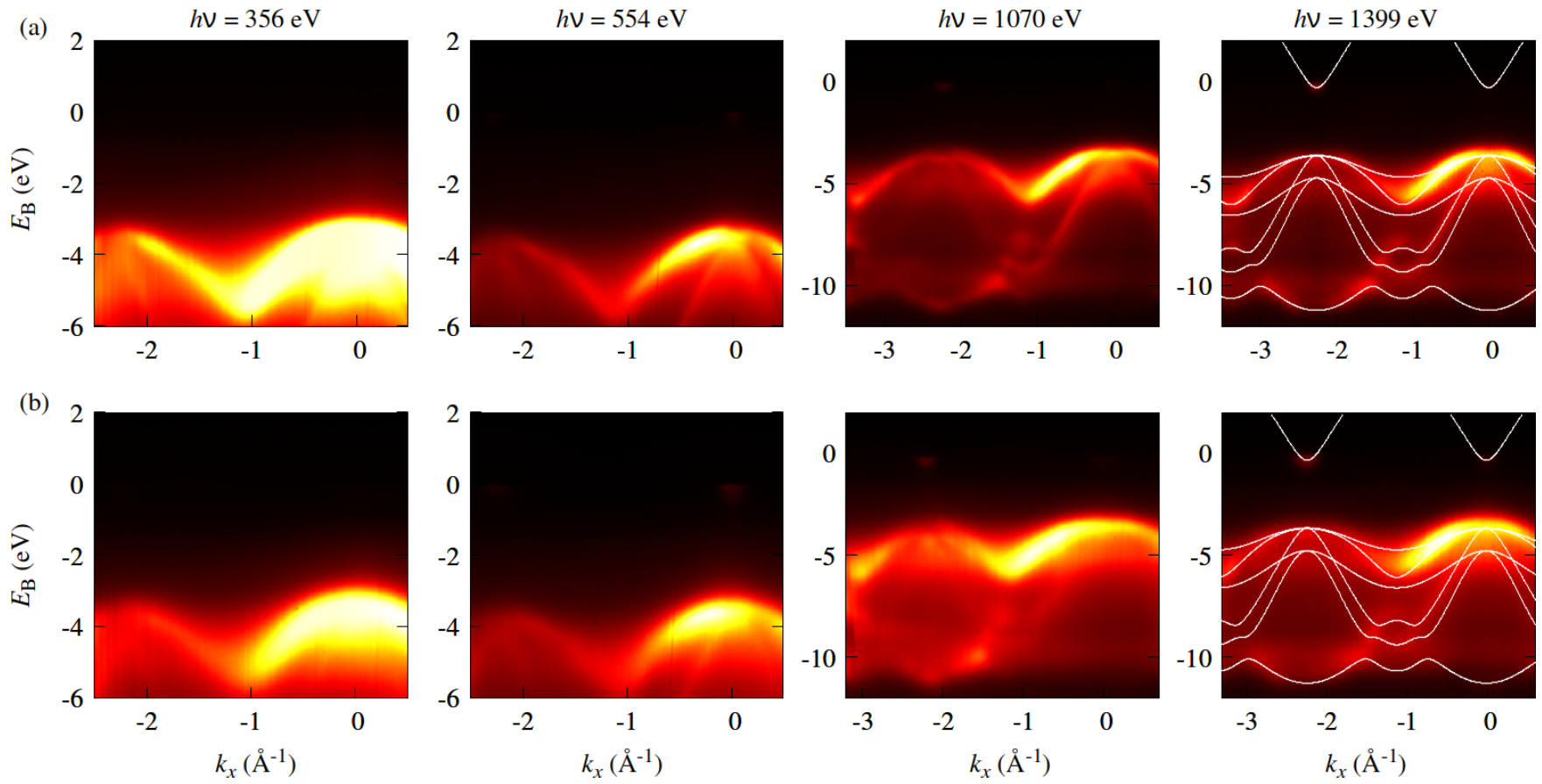

FIG. 2. Angle-resolved photoelectron spectroscopy (ARPES) dispersions for $\mathrm{GaN}$ : $\mathrm{Si}$ along the $\Gamma M$ directions of the bulk Brillouin zone (BZ) for (a) $n_{e}=8.3 \times 10^{19} \mathrm{~cm}^{-3}$ and (b) $n_{e}=1.5 \times 10^{20} \mathrm{~cm}^{-3}$. The $h v$ values are indicated (note different $E_{B}$ scales). The experimental dispersions broaden with increase of $n_{e}$ and sharpen with increase of $h v$. The data for $h v=1399 \mathrm{eV}$ is superimposed with calculated $E$ (k) (white lines, with adjusted bandgap value). The conduction band (CB) states appear above the valence band (VB) continuum.

cross-section of the valence states is extremely small [43]. To maximize the coherent spectral fraction, reduced by the Debye-Waller factor due to thermal atomic motion [38], the measurements were performed at $12 \mathrm{~K}$. The combined (beamline and analyzer) energy resolution varied from $60 \mathrm{meV}$ for $h v=350 \mathrm{eV}$ to $270 \mathrm{meV}$ for $h v=1400 \mathrm{eV}$, and the analyzer angular resolution was $\sim 0.1^{\circ}$. Other relevant details of the SX-ARPES experiment, including the experimental geometry, are identical to our previous study on GaN-HEMTs [44] and presented in Fig. 1(b). The binding-energy $\left(E_{B}\right)$ scale was referenced to the Fermi level $\left(E_{F}\right)$ in the $\mathrm{CB}$ of $\mathrm{GaN}$ : Si.

\section{Experimental results}

The use of SX energy range for our ARPES experiments on $\mathrm{GaN}$ : Si samples has been necessitated by a contaminated and partially disordered layer remnant at the sample surface after our ex situ sample transfer and preparation. Figure 2 shows the experimental ARPES dispersions of the VB/CB measured along the $\Gamma M$ direction of the bulk Brillouin zone (BZ), see Fig. 1(c), for two samples with Si-dopant concentrations of $n_{e}=8.3 \times 10^{19}$ and $1.5 \times 10^{20} \mathrm{~cm}^{-3}$. They were measured at $h v=356,554,1070$, and $1399 \mathrm{eV}$ which bring $k_{z}$ to the $\Gamma$ point as determined from the ARPES dispersions as a function of $h v$ (see below). The nondispersive spectral fraction, building up due to the thermal atomic motion and defect scattering, is suppressed in these plots by subtracting angle-integrated spectral intensity. We note that, because of the disorder induced by Si doping, the experimental dispersions broaden in energy with increase of $n_{e}$. The dispersions sharpen toward higher $h v$, even though the experimental energy resolution worsens, because the increase of $\lambda_{\mathrm{PE}}$ both reduces the relative spectral contribution from the near-surface region where the $V(z)$ variation is steepest and simultaneously sharpens the intrinsic $k_{z}$ resolution. The ARPES image at $h v=1399 \mathrm{eV}$ shows a fair agreement with the DFT calculations. The doped $\mathrm{CB}$ states appear as a tiny electron pocket above the VB dispersions. The energy separation between the VBM and $\mathrm{CBM}$ is consistent with the known GaN bandgap $\sim 3.3 \mathrm{eV}$. The CB intensity relative to the VB scales up with an increase of $h v$, which is apparent in the energy-distribution curves (EDCs) at the $\Gamma$ point in Fig. 3. This effect is attributed to the surface-induced band bending, discussed below, where the filling of the CB states, in contrast to the VB states, increases with the depth. In this case, the increase of $\lambda_{\mathrm{PE}}$ with $h v$ adds

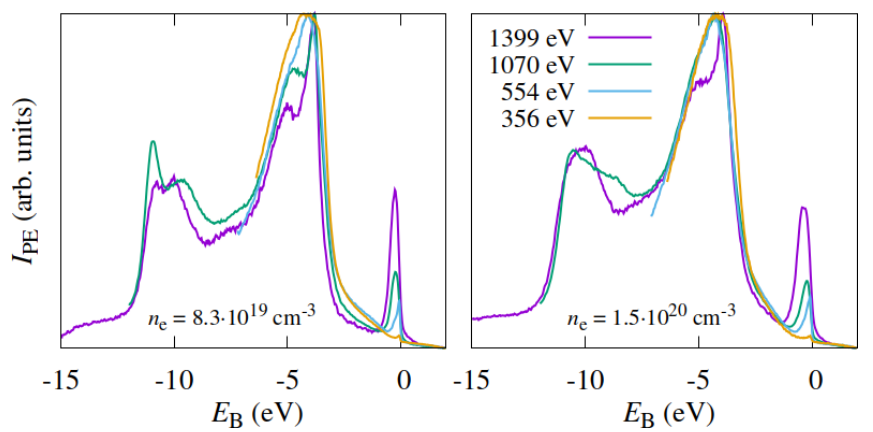

FIG. 3. Energy-distribution curve (EDC) spectra at the $\Gamma$ point measured at different $h v$ for $n_{e}=8.3 \times 10^{19} \mathrm{~cm}^{-3}$ and $1.5 \times$ $10^{20} \mathrm{~cm}^{-3}$. The conduction band (CB) signal scales up with $h v$ due to the surface band bending. 
(a) $\quad E_{\mathrm{B}}=-3.2 \mathrm{eV}$

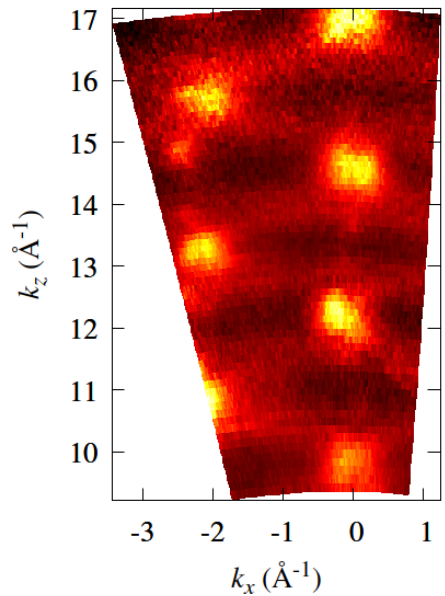

(b) Fermi surface

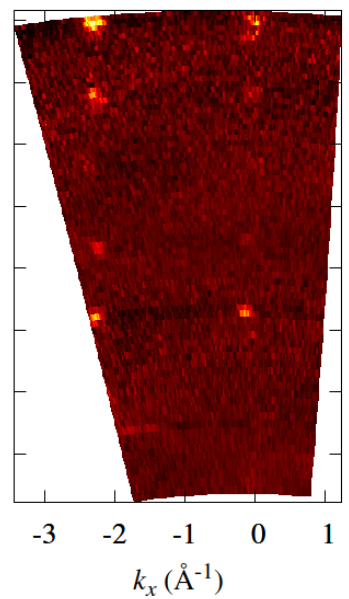

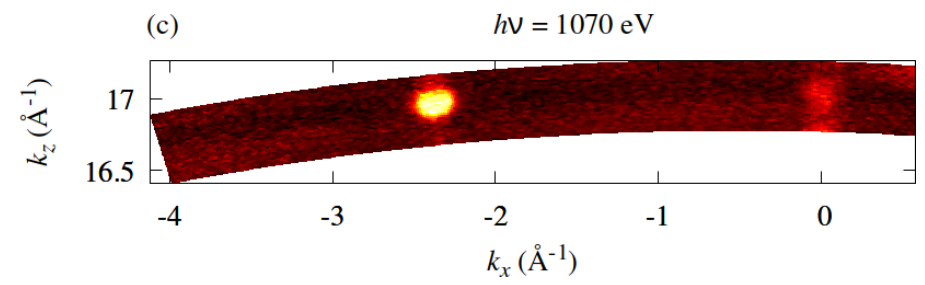

(d) $\quad h \mathrm{~V}=554 \mathrm{eV}$

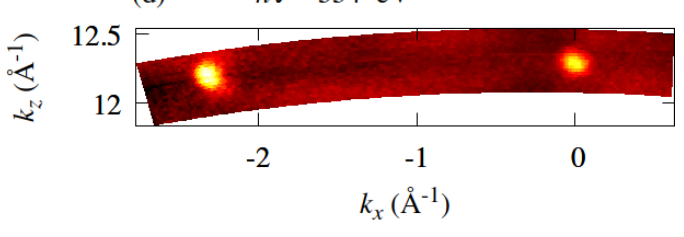

FIG. 4. $h v$-dependent angle-resolved photoelectron spectroscopy (ARPES) data for the sample with $n_{e}=6.4 \times 10^{19} \mathrm{~cm}^{-3}$ rendered into out-of-plane iso- $E_{B}$ maps in the $\Gamma A L M$ plane of the bulk Brillouin zone (BZ): (a) Valence band (VB) states near the VB maximum (VBM). (b) Fermi surface (FS) formed by the doped conduction band (CB) states. (c) and (d) Zoom-ins of the FS map measured for the sample with $n_{e}=8.3 \times 10^{19} \mathrm{~cm}^{-3}$ at $\sim 1070$ and $554 \mathrm{eV}$, respectively. The circular shape of the FS pockets confirms the three-dimensional (3D) character of the doped CB states.

signal from the progressively filling CB states in the sample depth.

We will now analyze the ARPES data as a function of $h v$, which controls the out-of-plane electron momentum $k_{z}$ (the conversion formulas for our experimental geometry, including the photon-momentum correction, are compiled in Ref. [24]). These data for one of the samples, measured in the $\Gamma M$ azimuth and rendered into an out-of-plane iso- $E_{B}$ map in $\left(k_{x}, k_{z}\right)$ coordinates in the $\Gamma A L M$ plane of the bulk BZ, see Fig. 1(c), are displayed in Figs. 4(a) and 4(b). The map corresponding to the VBM, displayed in (a), shows $k_{Z}$-dispersive contours characteristic of the 3D character of the VB states in $\mathrm{GaN}$ : $\mathrm{Si}$. The map corresponding to the Fermi level $\left(E_{F}\right)$, displayed in (b), reveals the Fermi surface (FS) of GaN : Si formed by electron doping into the CB. The hole pockets at the VBM and electron pockets at the CBM are centered at the $k_{z}$ values which are integers of $G_{z}$, i.e., correspond to the $\Gamma$ points of the reduced BZ. Measured in two different $h v$ regions, highresolution zoom-ins of these pockets in Figs. 4(c) and 4(d) show a circular shape. This pattern confirms the 3D nature of the doped CB states. The $h v$-dependent ARPES data from the GaN : Si samples with different $n_{e}$ were essentially identical, apart from the area of the FS pockets which increased with $n_{e}$.

Experimental in-plane iso- $E_{B}$ maps in $\left(k_{x}, k_{y}\right)$ coordinates measured for the sample with $n_{e}=8.3 \times 10^{19}$ are displayed in Fig. 5, the one corresponding to the VBM in (a) and the $\mathrm{CB}$-derived FS in (b). Acquired at $h v=1070 \mathrm{eV}$, they correspond to the $\Gamma K M$ plane of the bulk BZ, see Fig. 1(c). The FS image appears as tiny electron pockets around the $\Gamma$ points. High-resolution zoom-ins of this FS for three samples with $n_{e}=3.0 \times 10^{19}, 8.3 \times 10^{19}$, and $1.5 \times 10^{20} \mathrm{~cm}^{-3}$ measured at two different $h v$ values are shown in Fig. 5(c). Following the Luttinger theorem, which states that the FS volume is proportional to the charge-carrier concentration, the apparent area of the electron pockets increases with increase in $n_{e}$. Furthermore, we note that this area decreases with decrease of $h v$; as we will see below, this effect is due to the surface band bending.

\section{THEORETICAL ELECTRONIC STRUCTURE}

\section{A. Computational procedure}

First-principles calculations for bulk Si-doped GaN have been carried out in the DFT framework as implemented in the VASP code [45-47]. It has previously been demonstrated that the local-density approximation (LDA), generalized gradient approximation, and LDA $+U$ approximations all fail to reproduce the correct bandgap of $\mathrm{GaN}$ [48]. Hybrid functionals of the HSE-type would correct this drawback, but their computational cost is prohibitive for unit cells of a few hundreds of atoms typically used to model impurity systems. The LDA, however, correctly reproduces the band dispersions and $m^{*}$ for wurtzite $\mathrm{GaN}$, with $m^{*}=0.185 m_{0}$ in the CBM $\left(m_{0}\right.$ is the free-electron mass) closely matching the experimental $m^{*}=0.2 m_{0}$ [49]. Here, we will limit ourselves to spinless LDA calculations.

Self-consistent calculations were performed with a planewave cutoff energy of $400 \mathrm{eV}$ and $\mathbf{k}$-space sampling over a $\Gamma$-centered grid of $8 \times 8 \times 8$ points in the BZ. We used a supercell of $5 \times 5 \times 3$ unit cells of GaN [Fig. 6(a)]. We considered two types of impurity locations: substitutional and interstitial. In the first case, a $\mathrm{Ga}$ ion was substituted for a $\mathrm{Si}$ one. In the second case, a $\mathrm{Si}$ ion was placed to an interstitial position in the GaN lattice. The total number of ions was 300 and 301, respectively. The corresponding Si impurity concentration was $N_{\mathrm{D}}=2.9 \times 10^{20} \mathrm{~cm}^{-3}$. Atomic coordinates in the supercell were relaxed until the Hellmann-Feynman forces on each atom were $<30 \mathrm{meV}^{-1}$ under the constraint that the supercell shape and volume were the same as those of pure GaN. 
(a)

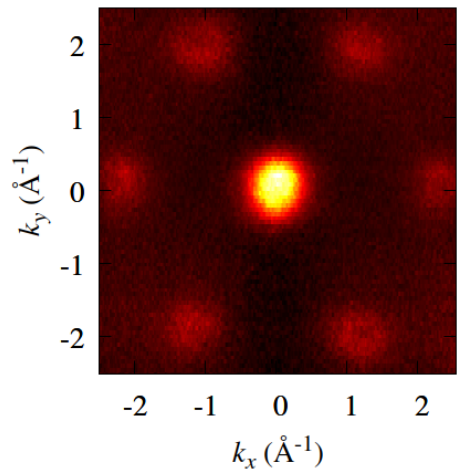

(b) $\quad n_{\mathrm{e}}=8.3 \cdot 10^{19} \mathrm{~cm}^{-3}$

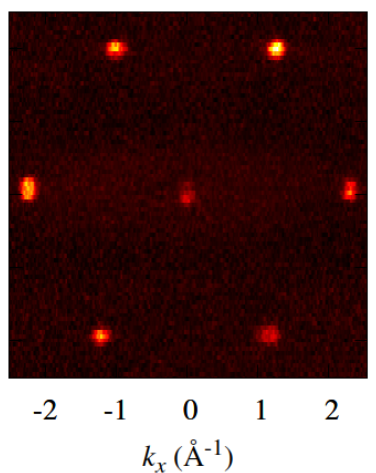

$h \mathrm{~V}=1070 \mathrm{eV}$
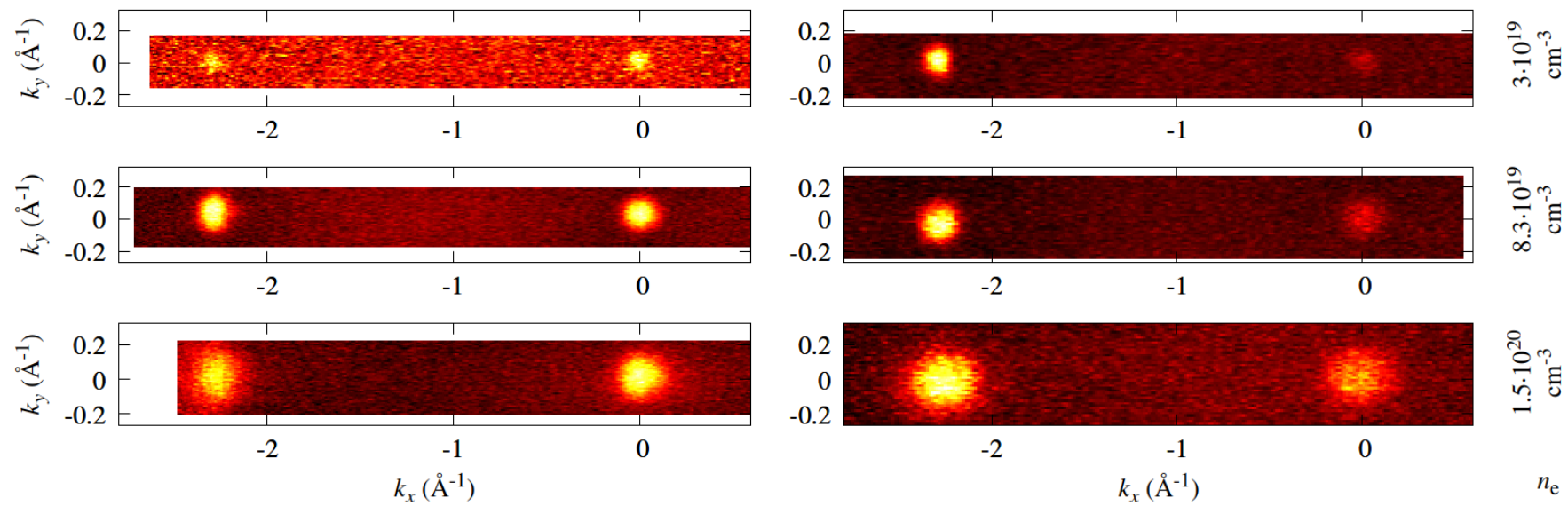

FIG. 5. In-plane iso- $E_{B}$ maps measured for the sample with $n_{e}=8.3 \times 10^{19} \mathrm{~cm}^{-3}$ : (a) Valence band (VB) states near the VB maximum (VBM; at $E_{B}=-3.3 \mathrm{eV}$ integrated over an energy window of $0.05 \mathrm{eV}$ ) and (b) Fermi surface (FS) formed by the doped conduction band (CB) states in the $\Gamma K M$ plane of the bulk Brillouin zone (BZ), measured at $h v=1070 \mathrm{eV}$. (c) Zoom-ins of the FS map measured for three samples with different $n_{e}$ and at two different $h v$ (indicated). The apparent area of the electron pockets increases with doping and decreases with decrease of $h v$ due to the surface band bending.

\section{B. Theoretical results \\ 1. Substitutional impurity}

The calculated band structure of $\mathrm{GaN}: \mathrm{Si}$ in the folded $\mathrm{BZ}$ and the orbital projections of $\mathrm{VB} / \mathrm{CB}$ states are presented in
Figs. 6(b) and 6(c). The circle radii are proportional to projection values $\left|\left\langle Y_{l m} \mid \varphi_{n \mathbf{k}}\right\rangle\right|^{2}$, where $\mathrm{Y}_{l m}$ is the spherical harmonic centered at the $\mathrm{Ga}, \mathrm{N}$, and $\mathrm{Si}$ ions, and $\varphi_{n \mathbf{k}}$ is the $\mathrm{VB} / \mathrm{CB}$ wave function for the band index $n$ and momentum $\mathbf{k}$. The (a)

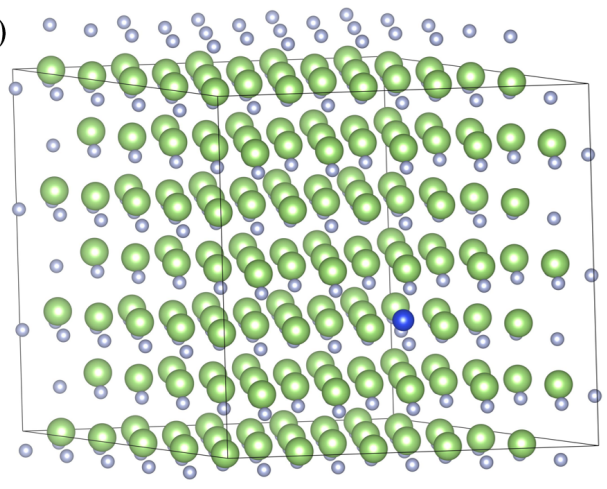

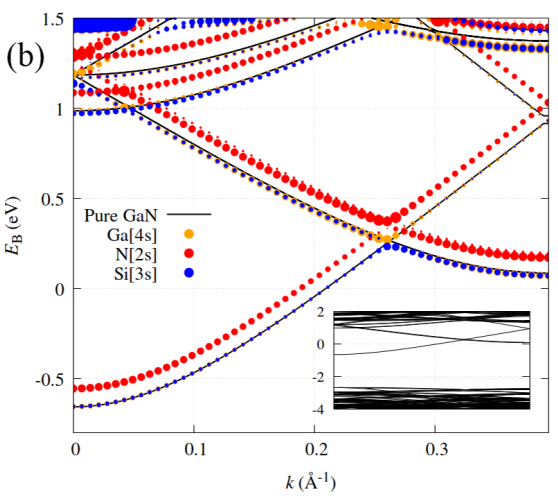

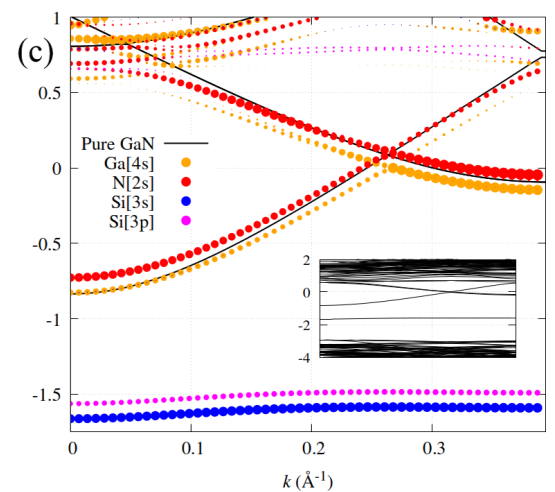

FIG. 6. Density functional theory (DFT) calculations for GaN : Si: (a) A supercell of $5 \times 5 \times 3$ unit cells of GaN with a substitutional Si impurity (blue ball) used in the calculations. Theoretical conduction band (CB) of $\mathrm{GaN}: \mathrm{Si}$ in the $\Gamma K$ direction for the (b) substitutional and (c) interstitial Si impurities. The orbital projections of $\mathrm{CB}$ states onto Ga $4 s, \mathrm{~N} 2 s, \mathrm{Si} 3 s$, and $\mathrm{Si} 3 p$ atomic orbitals are shown. For clarity, the N $2 s$ and Si $3 p$ projections are offset vertically by $0.1 \mathrm{eV}$. The electron dispersions in pure GaN are shown with the solid lines. While the substitutional Si impurity fully integrates into the GaN host electron system for the occupied states, the interstitial one forms a midgap state decoupled from the host. The inset shows the band structure of $\mathrm{GaN}: \mathrm{Si}$ in the energy range from -4 to $2 \mathrm{eV}$. 

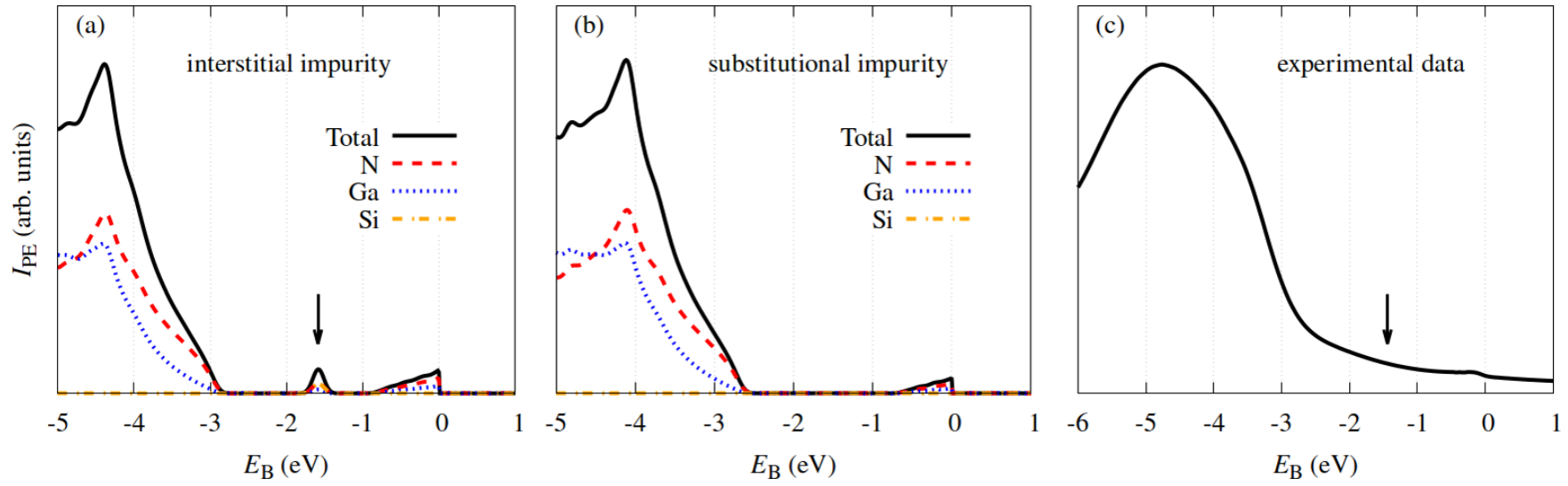

FIG. 7. Partial $I_{\mathrm{PE}}^{i j}\left(E_{B}\right)$ and total $I_{\mathrm{PE}}\left(E_{B}\right)$ angle-resolved photoelectron spectroscopy (ARPES) intensities simulated with $h v=1070 \mathrm{eV}$ for (a) interstitial and (b) substitutional Si impurity atoms, and (c) experimental angle-integrated spectrum. The arrows in (a) and (c) mark the calculated $E_{B} \sim-1.6 \mathrm{eV}$ of the interstitial impurity state, absent in the experiment.

projections for the $\mathrm{Ga}$ and $\mathrm{N}$ ions were computed for the ions located far from the Si impurity.

Near the CBM, the projections of $\mathrm{CB}$ states onto the $\mathrm{Ga}$ $4 s, \mathrm{~N} 2 s$, and $\mathrm{Si} 3 s$ atomic orbitals all have the same order of magnitude. This suggests that the CB is formed because of hybridization of the host and Si-impurity states. The calculations indicate that, for the occupied states, the substitutional Si impurity fully integrates into the host band structure without formation of any additional impurity states in the gap [Fig. 6(b)]. We find that $m^{*}$ at the CB bottom is isotropic in the (0001) plane, and its value $m^{*}=0.185 m_{0}$ is the same as in pure GaN. The Fermi level is located $\sim 0.65 \mathrm{eV}$ above the CBM. The Fermi momentum $k_{F}$ is isotropic in the (0001) plane, and its value is $0.21 \AA^{-1}$.

\section{Interstitial impurity}

Moving a Si-impurity ion into the interstitial position has a dramatic effect on the band structure, as shown Fig. 6(c). In contrast to the substitutional case, a narrow impurity band is clearly visible in the middle of the GaN bandgap at around a binding energy of $1.5 \mathrm{eV}$. The atomic-orbital projections show that this midgap state is dominated by the Si atomic orbitals at a negligible weight of the $\mathrm{Ga}$ and $\mathrm{N}$ ones. This identifies this state as the Si-impurity state electronically decoupled from the host GaN.

We have also performed similar DFT calculations with a larger $5 \times 5 \times 5$ supercell including 500/501 atoms. Two supercell configurations, with a single impurity atom $\left(N_{\mathrm{D}}=1.7 \times 10^{20} \mathrm{~cm}^{-3}\right)$ and with two randomly distributed ones $\left(N_{\mathrm{D}}=3.5 \times 10^{20} \mathrm{~cm}^{-3}\right)$, produced qualitatively the same results. As the final remark, we note that our calculations with the LDA exchange correlation may describe less accurately the impurity states localized due to the pair $e-e$ interaction within the Mott-transition picture [5].

\section{DISCUSSION}

\section{A. Bulk electronic structure: Nature of the Si doping}

We will now compare the theoretical electronic structure of bulk GaN : Si with the ARPES experiment to identify the preferential position of the $\mathrm{Si}$ impurities in the $\mathrm{GaN}$ host. At this stage, we neglect the surface band bending, whose effects are not critical for the following analysis of the bulk electronic structure.

To compare the DFT results with the ARPES data, we have also calculated partial element and orbital projected DOS (PDOS) for GaN with substitutional and interstitial Si impurities. Here, k-integrated ARPES intensity $I_{\mathrm{PE}}\left(E_{B}, h v\right)$ was simulated by summation of the partial intensities $I_{\mathrm{PE}}^{i j}\left(E_{B}, h v\right)$ from each element $i(=\mathrm{Ga}, \mathrm{N}$, and $\mathrm{Si})$ and orbital $j(=s$ and $p)$ evaluated as the PDOS contributions $\rho_{i j}(E)$ multiplied by the $h v$-dependent atomic photoionization cross-sections $\sigma_{i j}(h v)$ taken from Ref. [43]:

$$
\begin{aligned}
& I_{\mathrm{PE}}\left(E_{B}, h v\right)=\Sigma_{i} I_{\mathrm{PE}}^{i}\left(E_{B}, h v\right), \\
& I_{\mathrm{PE}}^{i}\left(E_{B}, h v\right) \sim \Sigma_{j} \sigma_{i j}(h v) \rho_{i j}\left(E_{B}\right) f_{\mathrm{FD}}\left(\frac{E_{B}-E_{F}}{T}\right),
\end{aligned}
$$

where $f_{\mathrm{FD}}$ is the Fermi-Dirac distribution. The partial $\rho_{i j}\left(E_{B}\right)$ were obtained from projections of $\varphi_{n \mathbf{k}}$ onto the spherical harmonics that are nonzero within spheres of a certain radius centered at the corresponding ions. A Gaussian smearing with an energy window width of $100 \mathrm{meV}$ was introduced into these calculations to account for the instrumental broadening.

The ARPES spectra simulated for $h v=1070 \mathrm{eV}$ with the interstitial and substitutional $\mathrm{Si}$ impurity atoms in $\mathrm{GaN}: \mathrm{Si}$ are presented in Figs. 7(a) and 7(b), respectively. The midgap peak in (a), absent in (b), marks the Si-derived impurity state. It has small but noticeable weight and, for $n_{e}$ of order of $10^{20} \mathrm{~cm}^{-3}$ and above, should in principle be observable in the experiment. The absence of such a peak in our angle-integrated ARPES data, Fig. 7(c), confirms that the substitutional impurities dominate our $\mathrm{GaN}$ : Si samples. This analysis of the ARPES data in comparison with the theoretical electronic structure is an identification of the positions of $\mathrm{Si}$ atoms in GaN hardly accessible with conventional techniques. Our results confirm the previous theoretical predictions, see Ref. [50-54]. 
(a) $n_{\mathrm{e}}=8.3 \cdot 10^{19} \mathrm{~cm}^{-3}$
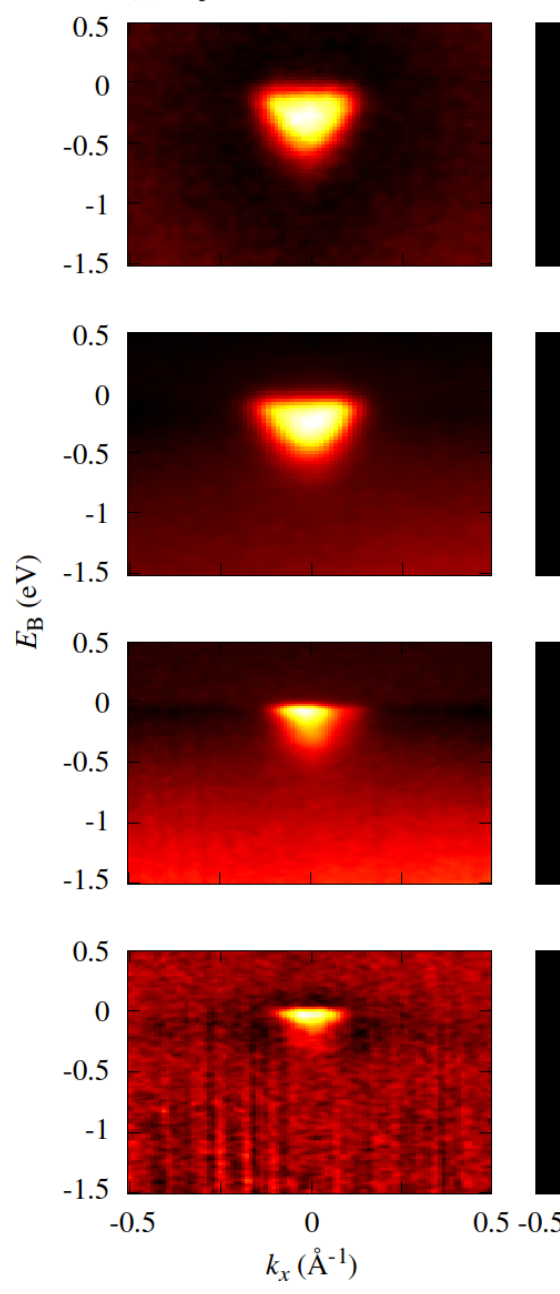
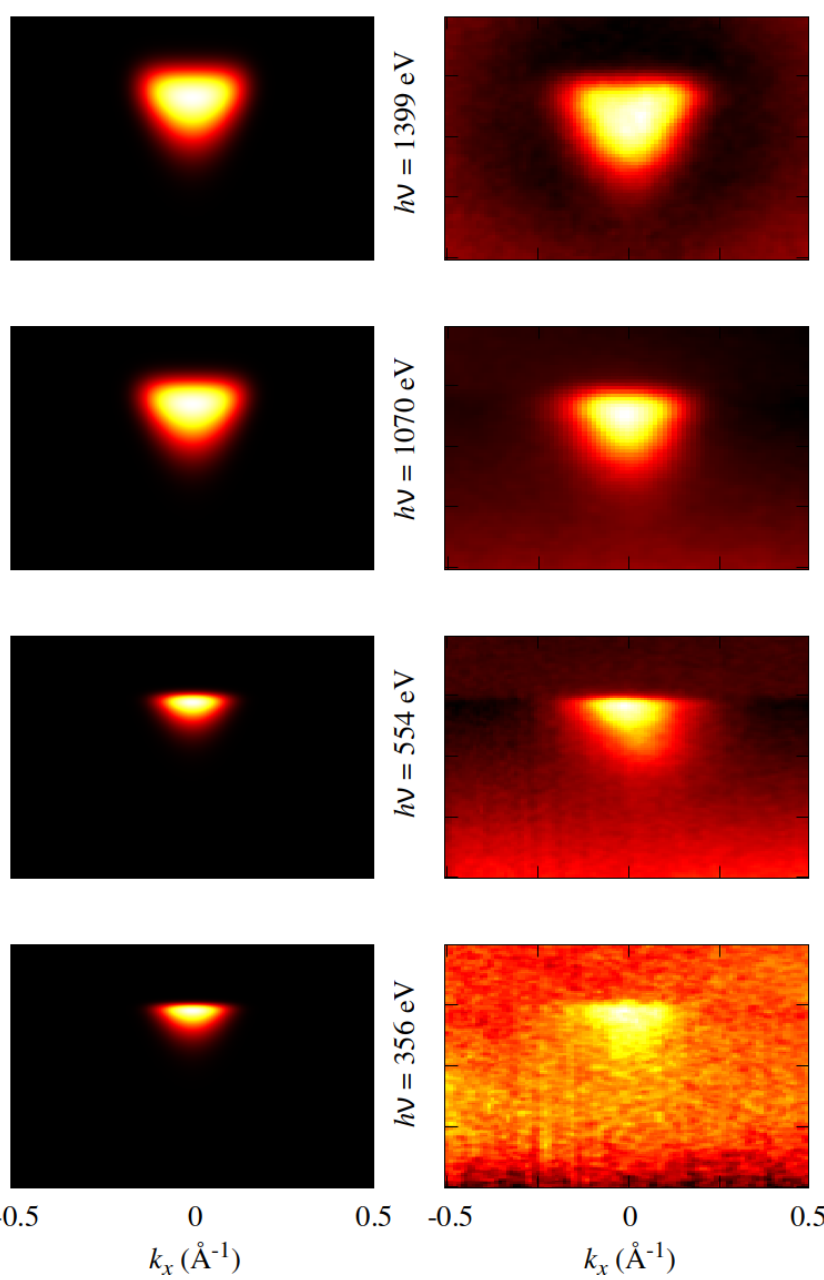

(b) $n_{\mathrm{e}}=1.5 \cdot 10^{20} \mathrm{~cm}^{-3}$
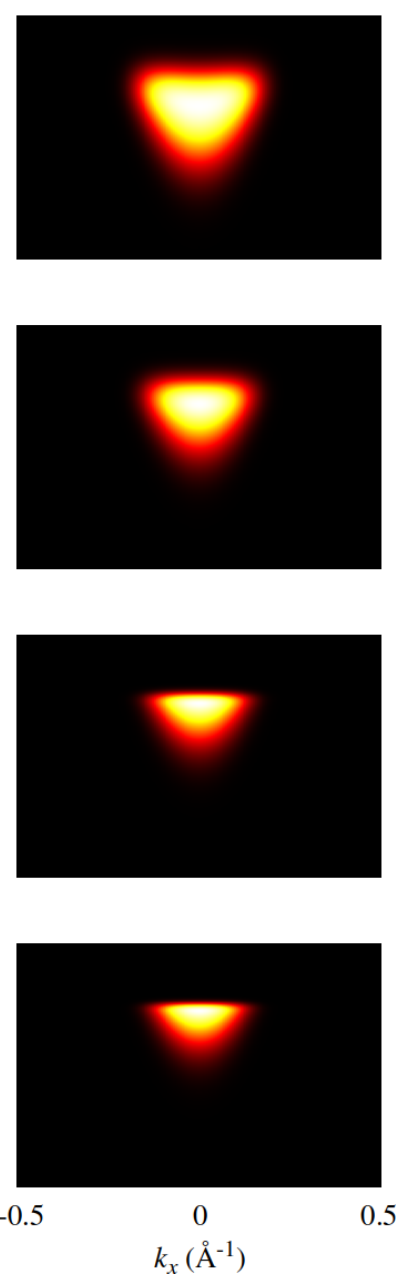

FIG. 8. Experimental (left column) and simulated (right) angle-resolved photoelectron spectroscopy (ARPES) images of conduction band (CB) for the systems with (a) $n_{e}=8.3 \times 10^{19} \mathrm{~cm}^{-3}$ and (b) $n_{e}=1.5 \times 10^{20} \mathrm{~cm}^{-3}$ and disorder-induced energy broadening $\delta_{\mathrm{D}}=0.2 \mathrm{eV} . \Lambda_{\mathrm{PE}}$ varies with $n_{e}$ and $h v$, as shown in Table I.

\section{B. Band-bending effects: Model and access to the bulk properties}

Band bending at semiconductor surfaces and interfaces forms quite a nontrivial electronic structure in this region. Its behavior can be approached following the ideas of the classical Wannier-Stark ladder formed in the electric field (see, for example, Ref. [55]). In this approach, the electronic structure of delocalized (valence and CB) states is formed by the hybridization of electron orbitals centered at each atomic site (electron hopping). The electric field offsets these orbitals in energy relative to each other, which reduces their hybridization. At a certain magnitude of the field, the orbitals completely decouple and can be considered as isolated electron states forming the Wannier-Stark ladder.

Nontrivial is also the response of such an electronic structure in the ARPES spectra. In simple terms, summation of the ARPES intensity throughout the band-bending region broadens and shifts the ARPES spectral peaks relative to the bulk states. Furthermore, relative contributions of the ARPES signals emerging at different depths will vary with the photoelectron mean free path $\lambda_{\mathrm{PE}}$, resulting in variation of the integral ARPES signal with $h v$. Such effects are immediately clear, for example, in our ARPES data from Figs. 4(c), 4(d), and 5(c), where the experimental FS appears not like a welldefined ring (such as the FS of the quantum well states in GaN-HEMTs [44]) but rather like a filled circle. Moreover, with an increase of $h v$ and the corresponding $\lambda_{\mathrm{PE}}$, the diameter of the circle increases. Another manifestation of the bandbending effect is the $h v$-dependent $\mathrm{CB}$ dispersions in Fig. 8, which are zoom-ins of the ARPES images from Fig. 2. We observe that the ARPES intensity there forms not a parabolic band (such as in GaN-HEMTs [44], even though somewhat blurred) but a filled parabola. Importantly, its extension in $E_{B}$ and $k_{x}$ clearly increases with $h v$. The filled-parabola image of the band dispersion and its extension in $E_{B}$ and $k_{x}$ depending on $h v$ are hallmarks of PE from bulk states in the band-bending region. While such effects for the VB states can be considered insignificant compared with the lifetime broadening (mostly due to $e$ - $e$ interaction, scaling up away from $E_{F}$ ), they become crucial for the much sharper states near the CBM. We note in passing that the surface or quantum well states do not change their energy through the band-bending 
region and therefore show a parabolic dispersion image independent of $h v[44,56]$.

Such distortive effects of the band bending on the ARPES signal do not allow direct determination of the FS and band dispersions in the GaN: Si bulk. We will now show that they can, however, be extracted by adequate modeling of the band-bending potential and corresponding ARPES response.

\section{Band-bending potential}

To compute the band-bending profile in the vicinity of the surface, we solve the quasi-one-dimensional Poisson and Schrödinger equations self-consistently [57]:

$$
\begin{aligned}
-\frac{\hbar^{2}}{2 m^{*}} \frac{d^{2} \psi_{n}(z)}{d z^{2}}+E_{c}(z) \psi_{n}(z) & =E_{n} \psi_{n}(z), \\
\frac{d}{d z}\left[\in \frac{d V(z)}{d z}\right] & =4 \pi\left[N_{\mathrm{D}}-n(z)\right],
\end{aligned}
$$

where $\psi_{n}(z)$ is the electron envelope function, $E_{c}(z)=$ $E_{c}(0)+V(z)$ the CB profile, $N_{\mathrm{D}}$ the concentration of ionized dopants, and

$$
n(z)=N_{c} \Sigma_{n}\left|\psi_{n}(z)\right|^{2} f_{\mathrm{FD}}^{(0)}\left(-\frac{E_{n}-E_{F}}{T}\right)
$$

is the local electron density in the CB. Here, $N_{c}=m^{*} T / \pi \hbar^{2}$ is the effective DOS in the $\mathrm{CB}, f_{\mathrm{FD}}^{(0)}(x)=\ln \left(1+e^{x}\right)$ is the zero-order Fermi-Dirac integral, which results from the twodimensional integration over the in-plane momentum $\left(k_{x}, k_{y}\right)$.

In the numerical implementation of this formalism, $m^{*}$ was taken from our DFT results. The surface potential $E_{c}(0)$, defined by the poorly controllable surface charge [58], is a free parameter of the model. Its value is adjusted for the best match between the ARPES simulations and experiment (see below). Here, $N_{\mathrm{D}}$ is assumed to be uniform in the whole system. The VB holes are treated within the quasiclassical Thomas-Fermi approximation because quantization of these states in the surface layer is expected to have only a small effect on the total electron-charge profile.

The $E_{c}(z)$ and $n(\mathrm{z})$ profiles calculated with different values $E_{c}(0)$ of the surface potential for the samples with the two largest $N_{\mathrm{D}}$ are shown in Fig. 9. Positive $E_{B}$ values near the surface reflect the formation of the surface depletion layer with vanishing electron concentration, whereas negative $E_{B}$ values deep in the sample correspond to the electron gas in the bulk. The $E_{c}(0)$ value is contributed by the negative polarization charge of the Ga polar surface and, possibly, by negatively charged surface states. The characteristic screening length is $\sim 3 \mathrm{~nm}$, this value being of the order of $\lambda_{\mathrm{PE}}$ in the ARPES experiment. Note that, whereas $N_{\mathrm{D}}$ is constant through the whole band-bending region, the local $n(z)$ varies and at large $z$ reaches its bulk electroneutrality asymptote $n_{e}=N_{\mathrm{D}}$.

\section{Simulation of the ARPES spectra}

Conventionally, the total PE spectrum $I_{\mathrm{PE}}\left(E_{B}\right)$ from localized states such as the CLs embedded in the band-bending potential $V(z)$ is described by summation of the intensity

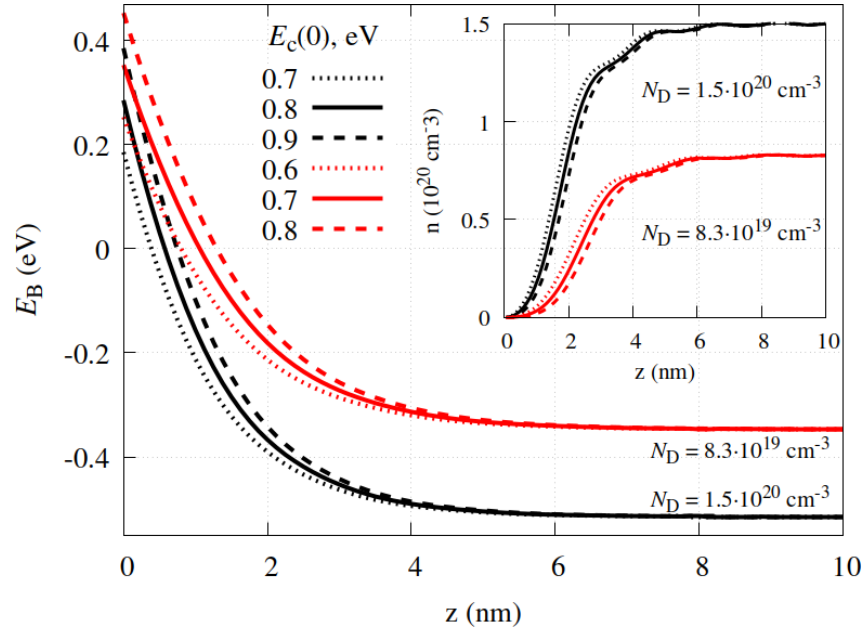

FIG. 9. The band profile and the electron concentration (inset) for different values of surface potential and dopant concentrations $N_{\mathrm{D}}=1.5 \times 10^{20} \mathrm{~cm}^{-3}$ (black lines) and $N_{\mathrm{D}}=8.3 \times 10^{19} \mathrm{~cm}^{-3}$ (red lines). The solid lines correspond to the parameters used for the simulation of the angle-resolved photoelectron spectroscopy (ARPES) spectra of the conduction band minimum (CBM).

contributions from all $i$ th atomic layers:

$$
I_{\mathrm{PE}}\left(E_{B}\right)=\Sigma_{i} \exp \left(-\frac{z_{i}}{\lambda_{\mathrm{PE}}}\right) I_{\mathrm{PE}}^{(i)}\left[E_{B}-V\left(z_{i}\right)\right],
$$

which are weighted by $\lambda_{\mathrm{PE}}$ and offset in energy from the bulk spectrum $I_{\mathrm{PE}}\left(E_{B}\right)$ by $V\left(z_{i}\right)$ (see, for example, Refs. [33,34,5961]). Such a summation returns an asymmetric profile of the resulting CL spectral line depending on $h v$ and emission angle, which can be used to extract the shape of $V(z)$ [35].

For the delocalized VB/CB states, this approach does not work, however, because the summation must consider not only the amplitudes but also phases of the wave functions. It can nevertheless be generalized to summation of amplitudes from the atomic layers-in the following, we will call this method the layer-summation (LS) one-provided that three conditions are met: (1) Like the CLs, the PE signal emanates mostly from the atomic-core region. This situation is characteristic of high-energy final states whose wave function rapidly oscillates. In this case, its scalar product with the $\mathrm{VB} / \mathrm{CB}$ wave function $\varphi(\mathbf{r})$, schematized in Fig. 10, averages out where $\varphi(\mathbf{r})$ smoothly varies between the atomic cores and survives only in the core regions [62] where $\varphi(\mathbf{r})$ rapidly oscillates due to the requirement of its orthogonality to the CLs. (2) Both VB/CB and final-state wave functions in different atomic planes are in phase, i.e., their phase accumulation across a layer $\exp \left(i k_{z} c\right)$, where $c$ is the out-of-plane lattice constant, is equal to one. This condition means that $k_{z} c=2 \pi n, n$ being an integer, i.e., $k_{z}$ matches one of the $\Gamma$ points of the extended BZ. (3) The sum includes not the intensities $I_{\mathrm{PE}}^{(i)}$ but the wave function amplitudes $\sim \sqrt{I_{\mathrm{PE}}^{(i)}}$ from each layer with the corresponding attenuation length $\lambda_{\mathrm{PE}} / 2$. The total sum is then obtained by squaring of the sum of the amplitudes:

$$
I_{\mathrm{PE}}\left(E_{B}\right)=\left\{\Sigma_{i} \exp \left(-\frac{2 z_{i}}{\lambda_{\mathrm{PE}}}\right) \sqrt{I_{\mathrm{PE}}^{(i)}\left[E_{B}-V\left(z_{i}\right)\right]}\right\}^{2} .
$$




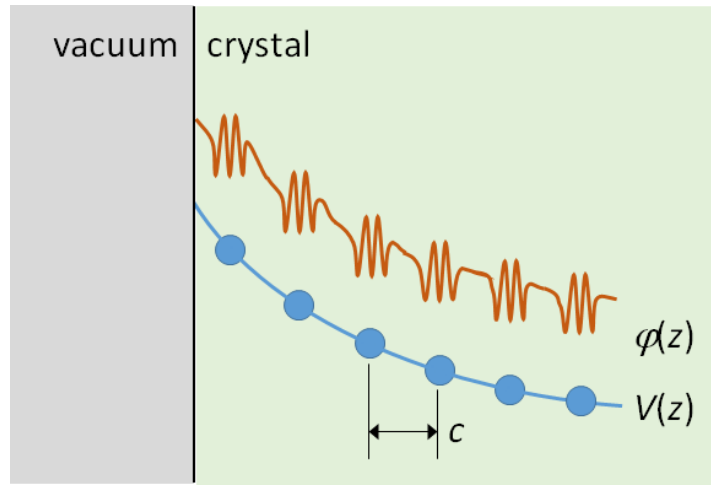

FIG. 10. Schematics of the potential $V(z)$ and valence band (VB)/conduction band (CB) wave function $\varphi(\mathbf{r})$ in the band-bending region in $\mathrm{Si}: \mathrm{GaN}$. The photoemission (PE) signal comes mostly from the atomic-core regions.

We note that a prerequisite for the LS method is the in-phase condition achieved at particular $k_{z}$ values tunable through $h v$. In general, this requires synchrotron-based x-ray sources because the laboratory $\mathrm{x}$-ray ones deliver only fixed $h v$.

Implementing this LS method for the CB states, we adopted their free-electron dispersion $E\left(k_{x}\right)=\hbar_{2} k_{x}^{2} / 2 m^{*}$ and described the ARPES spectrum as

$$
\begin{aligned}
I_{\mathrm{PE}}^{0}\left(k_{x}, E_{B}\right) \sim & f_{\mathrm{FD}}\left(\frac{E_{B}-E_{F}}{T}\right)\left\{\Sigma_{i} \exp \left(-\frac{2 z_{i}}{\lambda_{\mathrm{PE}}}\right)\right. \\
& \left.\times \sqrt{G_{\mathrm{D}}\left[E_{B}-E_{c}\left(z_{i}\right)-\frac{\hbar^{2} k_{x}^{2}}{2 m^{*}}\right]}\right\}^{2},
\end{aligned}
$$

where the Gaussians

$$
G_{\mathrm{D}}(E) \sim \exp \left[-\frac{E^{2}}{2\left(\delta_{\mathrm{D}} E\right)^{2}}\right]
$$

model the contribution $I_{\mathrm{PE}}^{(i)}$ of a single atomic layer at the position $z_{i}$ to the total $I_{\mathrm{PE}}$ for given $E_{B}$ and $k_{x}$. Here, the Gaussians account for the disorder and lifetime, which introduce an energy broadening of $\delta_{\mathrm{D}} E$. To account for instrumental angle $\left(\delta k_{x}\right)$ and energy $(\delta E)$ broadening, we further convolve $I_{\mathrm{PE}}^{0}\left(k_{x}, E_{B}\right)$ with two Gaussians:

$G\left(k_{x}\right) \sim \exp \left[-\frac{k_{x}^{2}}{2\left(\delta k_{x}\right)^{2}}\right]$ and $G(E) \sim \exp \left[-\frac{E^{2}}{2(\delta E)^{2}}\right]$,

to obtain the result:

$$
I_{\mathrm{PE}}\left(k_{x}, E_{B}\right) \sim \int d k_{x}^{\prime} d E^{\prime} I_{\mathrm{PE}}^{0}\left(k_{x}-k_{x}^{\prime}, E_{B}-E^{\prime}\right) G\left(k_{x}^{\prime}\right) G\left(E^{\prime}\right) .
$$

This summation procedure is illustrated in Fig. 11, which shows the partial $I_{\mathrm{PE}}^{(i)}$ spectra from a few layers along the above $V(\mathrm{z})$ simulated for $N_{\mathrm{D}}=1.5 \times 10^{20} \mathrm{~cm}^{-3}$. The weighted summation of these spectra will yield the filled-parabola pattern of the total $I_{\mathrm{PE}}\left(k_{x}, E_{B}\right)$ as seen in the experimental CB spectra, Fig. 8.

Our simulations of the CB spectra for different $N_{\mathrm{D}}$ used the corresponding $V(z)$ simulations above, with $m^{*}$ taken from the DFT calculations. Here, $\lambda_{\mathrm{PE}}$ was considered as a parameter

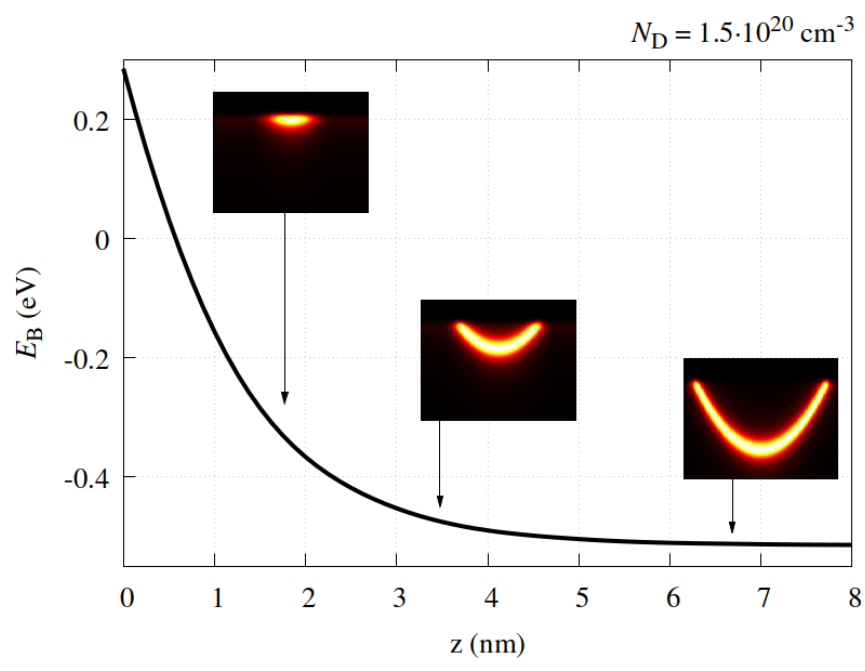

FIG. 11. The band-bending profile simulated for $N_{\mathrm{D}}=1.5 \times$ $10^{20} \mathrm{~cm}^{-3}$ and a schematic representation of the partial $I_{\mathrm{PE}}^{(i)}\left(k_{x}, E_{B}\right)$ from three atomic layers at different depths $z_{i}$. The summation of these spectra yields the filled-parabola pattern of the total $I_{\mathrm{PE}}\left(k_{x}, E_{B}\right)$.

that we varied until the simulated $I_{\mathrm{PE}}\left(k_{x}, E_{B}\right)$ fitted the experiment in that the apparent $k_{x}$ extent $2 \Delta k_{x}$ of the CB image at $E_{F}$, quantified by the extremes of the intensity gradient $d I_{\mathrm{PE}} / d k_{x}$, was equal to the corresponding experimental value. The simulated ARPES images are presented in Fig. 8 next to the corresponding experimental data. They adequately reproduce the experimental filled-parabola intensity pattern as well as the increase of its $\Delta k_{x}$ and energy depth with increase of $h v$ and $n_{e}$.

A summary of the ARPES experimental $\Delta k_{x}$ as a function of $h v$ and the corresponding results of the simulations are presented in Table I. It includes the two samples with maximal $n_{e}=1.5 \times 10^{20}$ and $8.3 \times 10^{19} \mathrm{~cm}^{-3}$ for which the $\Delta k_{x}$ values were well above the experimental $k_{x}$ resolution and could be determined with sufficient accuracy. The data obtained at the lowest $h v=356 \mathrm{eV}$ were excluded from the table as less reliable because of too small intensity of the $\mathrm{CB}$ signal in the experimental data.

The numerical results in Table I elucidate a few important points:

(1) As we have already seen in Fig. 8, the ARPES experimental $\Delta k_{x}$ values gradually increase with $h v$. This is a consequence of the corresponding increase of $\lambda_{\mathrm{PE}}$ at which the ARPES experiment probes the surface band-bending region in $\mathrm{GaN}$ : $\mathrm{Si}$.

(2) Table I also includes the bulk $k_{F}$ values determined from the magnetotransport $n_{e}$ through the Luttinger theorem [63-65]:

$$
n_{e}=2 \int_{\mathrm{FS}} \frac{d^{D} k_{F}}{(2 \pi)^{D}}
$$

where $D=3$ is the dimensionality of our system, and the integration is performed over the whole FS volume. We observe that, toward the high-energy end of our $h v$ range, the ARPES experimental $\Delta k_{x}$ values approach the bulk $k_{F}$. This observation brings up an important methodological aspect of the ARPES experiment on materials with surface band bending. 
TABLE I. Apparent $\Delta k_{x}$ extension of the CB electron pocket in the experimental ARPES spectra and the corresponding $\lambda_{\mathrm{PE}}$ values determined by simulations for two samples from the ARPES experiment having maximal $n_{e}$. The increase of $\Delta k_{x}$ with $h v$ manifests the band bending. The $\tau_{H}$ values derived from the Hall measurements are compared with the $\tau_{\mathrm{PE}}$ ones derived from $\lambda_{\mathrm{PE}}$.

\begin{tabular}{|c|c|c|c|c|c|c|c|c|}
\hline$n_{e}\left(\mathrm{~cm}^{-3}\right)$ & $k_{F}\left(\AA^{-1}\right)$ & $h v(\mathrm{eV})$ & $\Delta k_{x}\left(\AA^{-1}\right)$ & $E_{c}(0)(\mathrm{eV})$ & $\lambda_{\mathrm{PE}}(\mathrm{nm})$ & $\lambda_{\mathrm{TPP}-2 \mathrm{M}}(\mathrm{nm})$ & $\tau_{H}(\mathrm{fs})$ & $\tau_{\mathrm{PE}}(\mathrm{fs})$ \\
\hline \multirow{3}{*}{$(1.5 \pm 0.2) \times 10^{20}$} & $0.181 \pm 0.003$ & 1399 & $0.160 \pm 0.004$ & $0.8 \pm 0.1$ & $3.9 \pm 0.5$ & 3.25 & 9.5 & $0.063 \pm 0.01$ \\
\hline & & 1070 & $0.130 \pm 0.004$ & & $2.05 \pm 0.15$ & 2.64 & & $0.036 \pm 0.002$ \\
\hline & & 554 & $0.108 \pm 0.008$ & & $1.36 \pm 0.25$ & 1.62 & & $0.038 \pm 0.002$ \\
\hline \multirow{3}{*}{$(8.3 \pm 1.0) \times 10^{19}$} & $0.132 \pm 0.006$ & 1399 & $0.128 \pm 0.006$ & $0.7 \pm 0.1$ & $3.9 \pm 0.7$ & 3.25 & 10.9 & $0.063 \pm 0.01$ \\
\hline & & 1070 & $0.124 \pm 0.003$ & & $3.3 \pm 0.3$ & 2.64 & & $0.062 \pm 0.005$ \\
\hline & & 554 & $0.066 \pm 0.002$ & & $0.62 \pm 0.06$ & 1.62 & & $0.030 \pm 0.001$ \\
\hline
\end{tabular}

A naive use of the $\Delta k_{x}$ values apparent in low-energy ARPES spectra can be irrelevant in this case. To access the true bulk properties of the electronic structure, the ARPES experiment should use sufficiently high $h v$ where its probing depth exceeds the spatial extension of the band-bending region.

Another important factor that should be kept in mind in such an analysis is that the ARPES data in fact reflect local $n_{s}$ of the ordered metallic phase in the sample. The integral $n_{e}$ might in principle be smaller, with its difference compared with the ARPES value reflecting the volume fraction of the disordered or insulating phase [66]. This information allows assessment of spatial nonuniformity of the carrier distribution, which is a crucial property for the practical applications of $\mathrm{GaN}$ : Si. The fact that, in our case, the ARPES experimental $\Delta k_{x}$ values approach the $k_{F}$ determined from the magnetotransport $n_{e}$ evidences spatial homogeneity of our samples.

(3) The $\lambda_{\mathrm{PE}}$ values in Table I determined by fitting of our ARPES spectra appear quite close to those determined by the conventional CL-attenuation experiments on $\mathrm{GaN}[16,31,36]$ as well as to the values suggested by the conventional TPP$2 \mathrm{M}$ formula [67] as implemented in the NIST program [68] (also shown in Table I). Remarkably, the naive summation of the layer intensities rather than the wave function amplitudes returns $\lambda_{\mathrm{PE}}$ values which are underestimated by a factor of $\sim 2.5$.

(4) Finally, Table I compiles the photoelectron lifetimes ( $\left.\tau_{\mathrm{PE}}\right)$ determined from the $\lambda_{\mathrm{PE}}$ values as $\tau_{\mathrm{PE}}=\lambda_{\mathrm{PE}} / v_{\mathrm{PE}}$, where $v_{\mathrm{PE}}$ is the free-electron velocity in the crystal for given $E_{k}$. The $\tau_{H}$ values derived for our two samples from the Hall measurements are also included in Table I. They are more than two orders of magnitude larger than the $\tau_{\mathrm{PE}}$ ones. Indeed, whereas $\tau_{H}$ is limited only by the elastic $e-i$ (electron-impurity) scattering at $E_{F}, \tau_{\mathrm{PE}}$ is limited by much stronger inelastic $e-e$ and $e-i$ scattering (according to the wellknown Brooks-Herring formula [69], the elastic $e-i$ scattering strength reduces $\propto E_{k}{ }^{-3 / 2}$ and can therefore be neglected for photoelectrons). The observed increase of $\tau_{P E}$ with $E_{k}$ is consistent with the reduction of the inelastic $e-e$ and $e-i$ scattering with energy.

\section{CONCLUDING REMARKS}

Here, k-resolved one-electron band structure of heavily doped $\mathrm{GaN}: \mathrm{Si}$ with a wide range of $n_{e}$ has been determined, including electron dispersions throughout the VB/CB filled by the doped electrons. This fundamental knowledge is of key importance for the material properties of $\mathrm{GaN}: \mathrm{Si}$ widely used for nonalloyed ohmic contacts in modern GaN-based electronics. Crucial for our study has been the use of SX-ARPES whose enhanced probing depth has allowed access to the bulk electronic structure of $\mathrm{GaN}: \mathrm{Si}$ through its depleted surface band-bending region.

Comparison of the ARPES results with supercell DFT calculations for the interstitial and substitutional $\mathrm{Si}$ atoms in the GaN lattice has established the nature of $\mathrm{GaN}: \mathrm{Si}$ as a substitutional alloy, where Si preferentially substitutes for $\mathrm{Ga}$ and the doped electrons integrate into the whole electron system of $\mathrm{GaN}$ : $\mathrm{Si}$ without formation of an impurity band in the occupied states. This evidence achieved with SX-ARPES confirms the previous theoretical predictions and represents a fortunate simplification of the problem of Si-doped GaN.

With the sharp intrinsic resolution of our SX-ARPES experiment in the out-of-plane $k_{z}$, we have directly visualized the k-resolved electron dispersions throughout the VB/CB of $\mathrm{GaN}$ : Si. Apart from the known bandgap problem, they are well described by LDA-DFT band structure of bulk GaN . We have observed how the population of the CB states and expansion of the corresponding FS pockets follows the doped $n_{e}$. Puzzling peculiarities of the experimental ARPES spectra were the filled-parabola images of the CB dispersions as well as the dependence of the apparent $k_{F}$ on $h v$.

A quantitative analysis of the ARPES response of the CB states in $\mathrm{GaN}$ : Si requires, however, considering the surface band bending. We have put forward the insightful LS method to simulate this effect-a practical method to link the ARPES spectra with the evolution of bulk electron states through the band-bending region-where $V(z)$ is described via the self-consistent Poisson-Schrödinger equations, and the $\lambda_{\mathrm{PE}^{-}}$ weighted wave function amplitudes from the atomic layers are added up. This model relies on, first, high excitation energies whereby the ARPES intensity comes mostly from the atomiccore regions and, second, that the photoelectron $k_{z}$ matches out-of-plane reciprocal vectors whereby the layer contributions become in phase. The model can serve as a universal theoretical framework for any surface or interfacial crystalline system with band bending. It describes the filled-parabola image of the CB states in the GaN:Si ARPES spectra and the dependence of apparent $k_{F}$ on $h v$. At sufficiently high $h v$, where $\lambda_{\mathrm{PE}}$ exceeds the spatial extension of the bandbending region, the $k_{F}$ value apparent in the ARPES spectra 
approaches the bulk $k_{F}$ determined from magnetotransport $n_{e}$. This fact confirms the spatial homogeneity of our $\mathrm{GaN}: \mathrm{Si}$ samples without any notable admixture of an insulating fraction. Our simulation has allowed accurate determination of $E_{c}(0)$ - the band-bending height at the surface-as well as the $\lambda_{\mathrm{PE}}$ values, with the latter being markedly close to those predicted by the standard TPP-2M formula.

The knowledge about the k-resolved electronic structure of the band-bending region in $\mathrm{GaN}: \mathrm{Si}$ achieved in our exhaustive theoretical and experimental study is essential for understanding of the fundamental physics and theoretical modeling of the surface and interface systems in general and particularly of the HDSC-based systems such as nonalloyed ohmic contacts widely used in modern electronics.

\section{ACKNOWLEDGMENTS}

The SX-ARPES experiments were performed at the ADRESS beamline of the Swiss Light Source, Paul Scherrer Institute, Switzerland. The sample growth and characterization partially used the equipment of the Resource Center for Electrophysical Methods, and the computations the resources of the Federal Collective Usage Center "Complex for Simulation and Data Processing for Mega-science Facilities," both affiliated with the National Research Center "Kurchatov Institute" [70]. I.O.M., E.S.G., N.K.C., and V.G.V. were partially supported by the Russian Foundation for Basic Research under Grants No. 19-07-01090 and No. 20-0700813. N.B.M.S. was supported by the Microsoft Station Q Program.
[1] I. O. Maiboroda, A. A. Andreev, P. A. Perminov, Y. V. Fedorov, and M. L. Zanaveskin, Selective MBE growth of nonalloyed ohmic contacts to $2 \mathrm{D}$ electron gas in high-electron-mobility transistors based on GaN/AlGaN heterojunctions, Tech. Phys. Lett. 40, 488 (2014).

[2] B. Song, M. Zhu, Z. Hu, M. Qi, K. Nomoto, X. Yan, Y. Cao, D. Jena, and H. G. Xing, Ultralow-leakage AlGaN/GaN high electron mobility transistors on $\mathrm{Si}$ with non-alloyed regrown ohmic contacts, IEEE Electron Device Lett. 37, 16 (2016).

[3] T. Narita, K. Tomita, K. Kataoka, Y. Tokuda, T. Kogiso, H. Yoshida, N. Ikarashi, K. Iwata, M. Nagao, N. Sawada, M. Horita, J. Suda, and T. Kachi, Overview of carrier compensation in $\mathrm{GaN}$ layers grown by MOVPE: toward the application of vertical power devices, Jpn. J. Appl. Phys. 59, SA0804 (2020).

[4] B. I. Shklovskii and A. L. Efros, Electronic Properties of Doped Semiconductors (Springer, Berlin, Heidelberg, 1984), Vol. 45.

[5] N. Mott, Metal-Insulator Transitions (Taylor \& Francis, London, 1990).

[6] N. F. Mott, Metal-insulator transition, Rev. Mod. Phys. 40, 677 (1968).

[7] A. Wolos, Z. Wilamowski, M. Piersa, W. Strupinski, B. Lucznik, I. Grzegory, and S. Porowski, Properties of metalinsulator transition and electron spin relaxation in $\mathrm{GaN}: \mathrm{Si}$, Phys. Rev. B 83, 165206 (2011).

[8] V. F. Agekyan, E. V. Borisov, L. E. Vorobjev, G. A. Melentyev, H. Nykänen, L. Riuttanen, A. Yu. Serov, S. Suihkonen, O. Svensk, N. G. Filisofov, V. A. Shalygin, and L. A. Shelukhin, Optical and electrical properties of GaN : Si-based microstructures with a wide range of doping levels, Phys. Solid State 57, 787 (2015).

[9] E. Burstein, Anomalous optical absorption limit in InSb, Phys. Rev. 93, 632 (1954).

[10] T. S. Moss, The interpretation of the properties of indium antimonide, Proc. Phys. Soc. B 67, 775 (1954).

[11] S. Hofmann, Auger- and X-Ray Photoelectron Spectroscopy in Materials Science (Springer, Berlin, Heidelberg, 2013).

[12] M. Ladd and R. Palmer, Structure Determination by X-Ray Crystallography: Analysis by X-Rays and Neutrons (Springer Science \& Business Media, New York, 2013).

[13] J. P. Ibbetson, P. T. Fini, K. D. Ness, S. P. DenBaars, J. S. Speck, and U. K. Mishra, Polarization effects, surface states, and the source of electrons in $\mathrm{AlGaN} / \mathrm{GaN}$ heterostructure field effect transistors, Appl. Phys. Lett. 77, 250 (2000).

[14] V. M. Bermudez, The fundamental surface science of wurtzite gallium nitride, Surf. Sci. Rep. 72, 147 (2017).

[15] B. S. Eller, J. Yang, and R. J. Nemanich, Polarization effects of GaN and AlGaN: polarization bound charge, band bending, and electronic surface states, J. Electron. Mater. 43, 4560 (2014).

[16] Z. Huang, Y. Wu, Y. Zhao, L. Shi, R. Huang, F. Li, T. Liu, L. Xu, H. Gao, Y. Zhou, Q. Sun, S. Ding, K. Xu, and H. Yang, Accurate surface band bending determination on Ga-polar $n$-type GaN films by fitting $\mathrm{x}$-ray valence band photoemission spectrum, AIP Adv. 9, 115106 (2019).

[17] I. Bartoš, O. Romanyuk, T. Paskova, and P. Jiříček, Electron band bending and surface sensitivity: $\mathrm{x}$-ray photoelectron spectroscopy of polar GaN surfaces, Surf. Sci. 664, 241 (2017).

[18] D. Segev and C. G. Van de Walle, Electronic structure of nitride surfaces, J. Cryst. Growth 300, 199 (2007).

[19] J. H. Dycus, J. Houston Dycus, K. J. Mirrielees, E. D. Grimley, R. Kirste, S. Mita, Z. Sitar, R. Collazo, D. L. Irving, and J. M. LeBeau, Structure of ultrathin native oxides on III-nitride surfaces, ACS Appl. Mater. Interfaces 10, 10607 (2018).

[20] S. Sabuktagin, M. A. Reshchikov, D. K. Johnstone, and H. Morkoç, Band bending near the surface in $\mathrm{GaN}$ as detected by a charge sensitive probe, MRS Proceedings 798, 542 (2003).

[21] M. Feneberg, S. Osterburg, K. Lange, C. Lidig, B. Garke, R. Goldhahn, E. Richter, C. Netzel, M. D. Neumann, N. Esser, S. Fritze, H. Witte, J. Bläsing, A. Dadgar, and A. Krost, Band gap renormalization and Burstein-Moss effect in silicon- and germanium-doped wurtzite GaN up to $10^{20} \mathrm{~cm}^{-3}$, Phys. Rev. B 90, 075203 (2014).

[22] A. Damascelli, Z. Hussain, and Z.-X. Shen, Angle-resolved photoemission studies of the cuprate superconductors, Rev. Mod. Phys. 75, 473 (2003).

[23] C. S. Fadley, Looking deeper: angle-resolved photoemission with soft and hard x-rays, Synchrotron Radiat. News 25, 26 (2012).

[24] V. N. Strocov, X. Wang, M. Shi, M. Kobayashi, J. Krempasky, C. Hess, T. Schmitt, and L. Patthey, Soft-X-ray ARPES facility at the ADRESS beamline of the SLS: concepts, technical realisation and scientific applications, J. Synchrotron Rad. 21, 32 (2014). 
[25] V. N. Strocov, L. L. Lev, M. Kobayashi, C. Cancellieri, M.-A. Husanu, A. Chikina, N. B. M. Schröter, X. Wang, J. A. Krieger, and Z. Salman, k-resolved electronic structure of buried heterostructure and impurity systems by soft-x-ray ARPES, J. Electron Spectrosc. Relat. Phenom. 236, 1 (2019).

[26] V. N. Strocov, Intrinsic accuracy in 3-dimensional photoemission band mapping, J. Electron Spectrosc. Relat. Phenom. 130, 65 (2003).

[27] M. Kobayashi, I. Muneta, Y. Takeda, Y. Harada, A. Fujimori, J. Krempaský, T. Schmitt, S. Ohya, M. Tanaka, M. Oshima, and V. N. Strocov, Unveiling the impurity band induced ferromagnetism in the magnetic semiconductor (Ga,Mn)As, Phys. Rev. B 89, 205204 (2014).

[28] A. D. Bouravleuv, L. L. Lev, C. Piamonteze, X. Wang, T. Schmitt, A. I. Khrebtov, Y. B. Samsonenko, J. Kanski, G. E. Cirlin, and V. N. Strocov, Electronic structure of (In,Mn)As quantum dots buried in GaAs investigated by softx-ray ARPES, Nanotechnology 27, 425706 (2016).

[29] M. Kobayashi, L. D. Anh, J. Minár, W. Khan, S. Borek, P. N. Hai, Y. Harada, T. Schmitt, M. Oshima, A. Fujimori, M. Tanaka, and V. N. Strocov, Minority-spin impurity band in $n$-type (In,Fe)As: a materials perspective for ferromagnetic semiconductors, Phys. Rev. B 103, 115111 (2021).

[30] E. A. Kraut, R. W. Grant, J. R. Waldrop, and S. P. Kowalczyk, Semiconductor core-level to valence-band maximum bindingenergy differences: precise determination by x-ray photoelectron spectroscopy, Phys. Rev. B 28, 1965 (1983).

[31] Y. Zhao, H. Gao, R. Huang, Z. Huang, F. Li, J. Feng, Q. Sun, A. Dingsun, and H. Yang, Precise determination of surface band bending in Ga-polar n-GaN films by angular dependent x-ray photoemission spectroscopy, Sci. Rep. 9, 16969 (2019).

[32] S. Ueda, Depth-resolved electronic structure measurements by hard x-ray photoemission combined with x-ray total reflection: direct probing of surface band bending of polar GaN, Appl. Phys. Express 11, 105701 (2018).

[33] D. Wippler, R. G. Wilks, B. E. Pieters, S. J. van Albada, D. Gerlach, J. Hüpkes, M. Bär, and U. Rau, Pronounced surface band bending of thin-film silicon revealed by modeling core levels probed with hard x-rays, ACS Appl. Mater. Interfaces 8, 17685 (2016).

[34] Y. Du, P. V. Sushko, S. R. Spurgeon, M. E. Bowden, J. M. Ablett, T.-L. Lee, N. F. Quackenbush, J. C. Woicik, and S. A. Chambers, Layer-resolved band bending at the $n$ - $\mathrm{SrTiO}_{3}(001) / p$-Ge(001) interface, Phys. Rev. Materials 2, 094602 (2018).

[35] S. Schuwalow, N. B. M. Schröter, J. Gukelberger, C. Thomas, V. Strocov, J. Gamble, A. Chikina, M. Caputo, J. Krieger, G. C. Gardner, M. Troyer, G. Aeppli, M. J. Manfra, and P. Krogstrup, Band structure extraction at hybrid narrow-gap semiconductormetal interfaces, Adv. Sci. 8, 2003087 (2020).

[36] R. Huang, T. Liu, Y. Zhao, Y. Zhu, Z. Huang, F. Li, J. Liu, L. Zhang, S. Zhang, A. Dingsun, and H. Yang, Angular dependent XPS study of surface band bending on Ga-polar $n$-GaN, Appl. Surf. Sci. 440, 637 (2018).

[37] T. L. Duan, J. S. Pan, and D. S. Ang, Investigation of surface band bending of Ga-face GaN by angle-resolved x-ray photoelectron spectroscopy, ECS J. Solid State Sci. Technol. 5, P514 (2016).

[38] J. Braun, J. Minár, S. Mankovsky, V. N. Strocov, N. B. Brookes, L. Plucinski, C. M. Schneider, C. S. Fadley, and H. Ebert,
Exploring the XPS limit in soft and hard x-ray angle-resolved photoemission using a temperature-dependent one-step theory, Phys. Rev. B 88, 205409 (2013).

[39] D. M. Szmyd, M. C. Hanna, and A. Majerfeld, Heavily doped GaAs : Se. II. Electron mobility, J. Appl. Phys. 68, 2376 (1990).

[40] I. Halidou, Z. Benzarti, Z. Chine, T. Boufaden, and B. El Jani, Heavily silicon-doped GaN by MOVPE, Microelectron. J. 32, 137 (2001).

[41] K. Ueno, T. Fudetani, Y. Arakawa, A. Kobayashi, J. Ohta, and H. Fujioka, Electron transport properties of degenerate $n$-type GaN prepared by pulsed sputtering, APL Mater. 5, 126102 (2017).

[42] V. N. Strocov, T. Schmitt, U. Flechsig, T. Schmidt, A. Imhof, Q. Chen, J. Raabe, R. Betemps, D. Zimoch, J. Krempasky, X. Wang, M. Grioni, A. Piazzalunga, and L. Patthey, HighResolution soft x-ray beamline ADRESS at the Swiss Light Source for resonant inelastic $\mathrm{x}$-ray scattering and angleresolved photoelectron spectroscopies, J. Synchrotron Rad. 17, 631 (2010).

[43] J. J. Yeh and I. Lindau, Atomic subshell photoionization cross sections and asymmetry parameters: $1 \leqslant Z \leqslant 103$, At. Data Nucl. Data Tables 32, 1 (1985).

[44] L. L. Lev, I. O. Maiboroda, M.-A. Husanu, E. S. Grichuk, N. K. Chumakov, I. S. Ezubchenko, I. A. Chernykh, X. Wang, B. Tobler, T. Schmitt, M. L. Zanaveskin, V. G. Valeyev, and N. V. Strocov, k-space imaging of anisotropic 2D electron gas in GaN/GaAlN high-electron-mobility transistor heterostructures, Nat. Commun. 9, 2653 (2018).

[45] G. Kresse and J. Hafner, Ab initio molecular dynamics for liquid metals, Phys. Rev. B 47, 558 (1993).

[46] G. Kresse and J. Furthmüller, Efficiency of ab initio total energy calculations for metals and semiconductors using a plane-wave basis set, Comput. Mater. Sci. 6, 15 (1996).

[47] G. Kresse and J. Furthmüller, Efficient iterative schemes for $a b$ initio total-energy calculations using a plane-wave basis set, Phys. Rev. B 54, 11169 (1996).

[48] M. Chu, Characterization and modeling of strained Si FET and GaN HEMT devices, Ph.D. dissertation, University of Florida, 2011.

[49] V. Bougrov, M. E. Levinshtein, S. L. Rumyantsev, and A. Zubrilov, in Properties of Advanced Semiconductor Materials: GaN, AIN, InN, BN, SiC, SiGe, edited by M. E. Levinshtein, S. L. Rumyantsev, and M. S. Shur (John Wiley \& Sons, Inc., New York, 2001), pp. 1-30.

[50] J. Neugebauer and C. G. Van de Walle, Native defects and impurities in GaN, in Advances in Solid State Physics (Springer, Berlin, Heidelberg, 1996), Vol. 35, pp. 25-44.

[51] C. H. Park and D. J. Chadi, Stability of deep donor and acceptor centers in GaN, AlN, and BN, Phys. Rev. B 55, 12995 (1997).

[52] P. Bogusławski and J. Bernholc, Doping properties of C, Si, and Ge impurities in GaN and AlN, Phys. Rev. B 56, 9496 (1997).

[53] L. Gordon, J. L. Lyons, A. Janotti, and C. G. Van de Walle, Hybrid functional calculations of $D X$ centers in AlN and GaN, Phys. Rev. B 89, 085204 (2014).

[54] Z. Xie, Y. Sui, J. Buckeridge, C. R. A. Catlow, T. W. Keal, P. Sherwood, A. Walsh, D. O. Scanlon, S. M. Woodley, and A. A. Sokol, Demonstration of the donor characteristics of Si and O defects in GaN using hybrid QM/MM, Phys. Status Solidi A 214, 1600445 (2016). 
[55] E. E. Mendez and G. Bastard, Wannier-Stark ladders and Bloch oscillations in superlattices, Phys. Today 46(6), 34 (1993).

[56] V. N. Strocov, Photoemission response of 2D electron states, J. Electron Spectrosc. Relat. Phenom. 229, 100 (2018).

[57] A. Trellakis, A. T. Galick, A. Pacelli, and U. Ravaioli, Iteration scheme for the solution of the two-dimensional SchrödingerPoisson equations in quantum structures, J. Appl. Phys. 81, 7880 (1997).

[58] K. Köhler, J. Wiegert, H. P. Menner, M. Maier, and L. Kirste, The surface potential of GaN : Si, J. Appl. Phys. 103, 023706 (2008).

[59] H. Tanaka, I. Satoh, T. Kanki, E. Ikenaga, M. Kobata, J. J. Kim, S. Ueda, and K. Kobayashi, Nondestructive estimation of depletion layer profile in $\mathrm{Nb}$-doped $\mathrm{SrTiO}_{3} /(\mathrm{La}, \mathrm{Ba}) \mathrm{MnO}_{3}$ heterojunction diode structure by hard x-ray photoemission spectroscopy, Appl. Phys. Lett. 98, 133505 (2011).

[60] Y. Matveyev, D. Negrov, A. Chernikova, Y. Lebedinskii, R. Kirtaev, S. Zarubin, E. Suvorova, A. Gloskovskii, and A. Zenkevich, Effect of polarization reversal in ferroelectric $\mathrm{TiN} / \mathrm{Hf}_{0.5} \mathrm{Zr}_{0.5} \mathrm{O}_{2} / \mathrm{TiN}$ devices on electronic conditions at interfaces studied in operando by hard x-ray photoemission spectroscopy, ACS Appl. Mater. Interfaces 9, 43370 (2017).

[61] Y. Matveyev, V. Mikheev, D. Negrov, S. Zarubin, A. Kumar, E. D. Grimley, J. M. LeBeau, A. Gloskovskii, E. Y. Tsymbal, and A. Zenkevich, Polarization-dependent electric potential distribution across nanoscale ferroelectric $\mathrm{Hf}_{0.5} \mathrm{Zr}_{0.5} \mathrm{O}_{2}$ in functional memory capacitors, Nanoscale 11, 19814 (2019).

[62] C. Solterbeck, W. Schattke, J.-W. Zahlmann-Nowitzki, K.-U. Gawlik, L. Kipp, M. Skibowski, C. S. Fadley, and M. A. Van
Hove, Energetic and Spatial Bonding Properties from Angular Distributions of Ultraviolet Photoelectrons: Application to the GaAs(110) Surface, Phys. Rev. Lett. 79, 4681 (1997).

[63] J. M. Luttinger, Fermi surface and some simple equilibrium properties of a system of interacting fermions, Phys. Rev. 119, 1153 (1960).

[64] A. Praz, J. Feldman, H. Knörrer, and E. Trubowitz, A proof of Luttinger's theorem, EPL 72, 49 (2005).

[65] V. Brouet, M. Marsi, B. Mansart, A. Nicolaou, A. TalebIbrahimi, P. Le Fèvre, F. Bertran, F. Rullier-Albenque, A. Forget, and D. Colson, Nesting between hole and electron pockets in $\mathrm{Ba}\left(\mathrm{Fe}_{1-x} \mathrm{Co}_{x}\right)_{2} \mathrm{As}_{2}(x=0-0.3)$ observed with angle-resolved photoemission, Phys. Rev. B 80, 165115 (2009).

[66] V. N. Strocov, A. Chikina, M. Caputo, M.-A. Husanu, F. Bisti, D. Bracher, T. Schmitt, F. Miletto Granozio, C. A. F. Vaz, and F. Lechermann, Electronic phase separation at $\mathrm{LaAlO}_{3} / \mathrm{SrTiO}_{3}$ interfaces tunable by oxygen deficiency, Phys. Rev. Materials 3, 106001 (2019).

[67] S. Tanuma, C. J. Powell, and D. R. Penn, Calculation of electron inelastic mean free paths (IMFPs) VII. Reliability of the TPP2M IMFP predictive equation, Surf. Interface Anal. 35, 268 (2003).

[68] NIST Standard Reference Database 71, https://www.nist.gov/ srd/nist-standard-reference-database-71.

[69] D. Chattopadhyay and H. J. Queisser, Electron scattering by ionized impurities in semiconductors, Rev. Mod. Phys. 53, 745 (1981).

[70] http://ckp.nrcki.ru. 